\title{
ANÁLISIS LOCACIONAL DEL YACIMIENTO PALEOLÍTICO DE COVA EIRÓS (TRIACASTELA, LUGO)
}

\author{
LOCATIONAL ANALYSIS OF THE PALAEOLITHIC SITE OF COVA EIRÓS \\ (TRIACASTELA, LUGO)
}

MIKEL DÍAZ-RODRÍGUEZ ${ }^{(1)}$, RAMÓN FÁBREGAS-VALCARCE ${ }^{(1)}$, ARTURO DE LOMBERA-HERMIDA $^{(1)}$ \& XOSÉ PEDRO RODRÍGUEZ-ÁLVAREZ ${ }^{(2)(3)}$

Resumen: En este estudio presentamos el análisis locacional del yacimiento de Cova Eirós (Triacastela, Lugo). Se trata de un
lugar arqueológico que ha sido ocupado desde el Paleolítico medio hasta la actualidad. A partir de los SIG y la
estadística pretendemos acercarnos a aquellos factores medioambientales que definen su importancia como lugar de
ocupación a lo largo del tiempo y de manera recurrente. Una vez analizadas las variables que podrían marcar su patrón
de ocupación, hemos podido comprobar que Cova Eirós es un punto importante, destacado y estratégico, pues se halla
muy próximo a las rutas de tránsito potencial y con gran visibilidad. Además se encuentra resguardado y emplazado
sobre una fuerte pendiente. El yacimiento cuenta con recursos cinegéticos y zonas de obtención de materias primas
susceptibles de ser explotados en las inmediaciones, por lo que se trata de un enclave que presenta unas condiciones de
habitabilidad ideales para los grupos de cazadores-recolectores.

Palabras clave: SIG, Paleolítico, Análisis locacional, Patrón de ocupación, Noroeste Peninsular.

Abstract:

\begin{abstract}
In this paper, we deal with the locational analysis of the Cova Eirós site (Triacastela, Lugo), occupied from the Middle Palaeolithic to the present. From GIS and statistics, we intend to approach those environmental factors that define its importance as a place of occupation over time and on a recurring basis. Once we have analysed the variables that characterize the site's pattens of use, we have veriffed that Cova Eirós is an important, prominent and strategic point. characterize the site's patterns of use, we have verified that Cova Eiros is an important, prominent and The place is very close to the potential transit routes and has great visibility. It is also protected and set on a steep slope. Moreover the site has hunting resources and raw materials sources nearby. So it is a settlement that presents ideal living conditions for hunter-gatherer groups.
\end{abstract}

Keywords: GIS, Paleolithic, Locational analysis, Occupation pattern, Iberian Northwest.

Received: 14 January, 2020; Accepted: 7 January, 2021

\section{INTRODUCCIÓN}

En este trabajo se presenta el análisis de la localización del yacimiento de Cova Eirós. La elaboración de este estudio, nace de la hipótesis de que la elección de un emplazamiento determinado, como lugar de asentamiento por parte de las sociedades de cazadores-recolectores, responde a una decisión consciente e inclinada a satisfacer unas necesidades específicas y concretas. Para aproximarnos a esta problemática y para intentar responderla se utilizará una metodología específica, el análisis locacional. Éste se basa en la aplicación de los Sistemas de Información Geográfica (en adelante SIG), que nos permiten gestionar datos espaciales que serían inabarcables de otra forma. De esta manera, podemos aproximarnos al territorio de las comunidades del pasado y comprobar si la ocupación dilatada y continuada en el tiempo, documentada en Cova Eirós, es debida a su localización concreta y a las posibilidades que ofrece el territorio circundante o no.

La finalidad del análisis locacional es identi- ficar en qué medida los yacimientos arqueológicos se encuentran en el lugar en el que están ubicados porque sus pobladores han tomado ciertas decisiones o criterios de índole espacial. Los mismos no son accesibles a través del estudio de los elementos materiales arqueológicos, por lo que tenemos que intentar aproximarnos a través de determinados factores o variables locacionales para intentar comprobar en qué medida se priorizan unos sobre otros (Parcero OubiÑa \& Fábrega Álvarez 2006: 74). Generalmente estas variables tienden a ser ambientales, ya que son las más sencillas de modelizar. Es más complicado poder incorporar a un SIG otro tipo de variables, sean de índole social, cultural o política.

En este trabajo, nuestro objeto de estudio es un yacimiento, que posee ciertos criterios que pudieron condicionar las decisiones tomadas para emplearlo como lugar de asentamiento. Estos criterios están condicionados por determinados factores, que a su vez se concretan en decisiones. Estas decisiones locacionales son las que permiten establecer el modelo de análisis locacional (Fig. 1).

\footnotetext{
${ }^{(1)}$ Grupo de Estudos para a Prehistoria do Noroeste Ibérico-Arqueoloxía, Antigüidade e Territorio (GEPN-AAT). Universidade de Santiago de Compostela,

España. Autor de correspondencia: mikel.diaz.rodriguez@gmail.com
(2)Universitat Rovira i Virgili, Departament d'Història i Història de l'Art, Tarragona, España.

(3)Institut Català de Paleoecologia Humana i Evolució Social (IPHES-CERCA), Tarragona, España.
} 


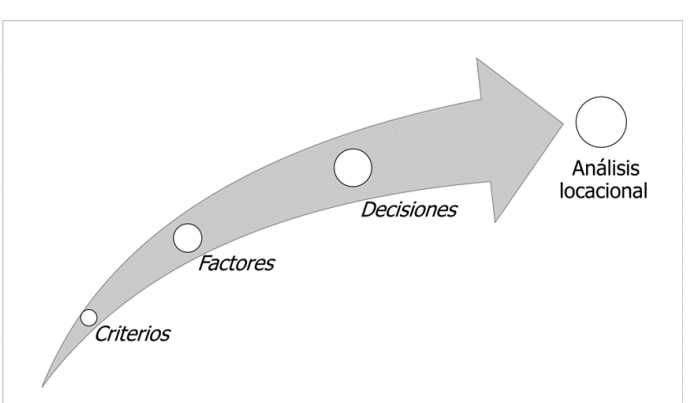

Fig. 1. Construcción del modelo teórico del análisis locacional. Fig. 1. Making up the theoretical model of location analysis.

A la hora de aplicar la metodología fundamentada en el análisis locacional, hay que tener en cuenta que algunas de las condiciones geográficas variaron a lo largo del tiempo, por lo que tenemos que seleccionar aquellas que permanecieron inamovibles o cuyos cambios no fueron sustanciales. Una vez definidos los criterios que llevaron a los cazadores-recolectores a asentarse en Cova Eirós, basándonos en modelos teóricos previos, podemos caracterizarlos mediante factores, fundamentalmente ambientales, para comprobar si se concretan en decisiones locacionales o no. El resultado nos permitirá establecer si esas variables influyeron en la localización del yacimiento y si, por consiguiente, incidieron en las decisiones sociales tomadas para establecerse en esta cavidad.

\section{HISTORIA DE LAS INVESTIGACIONES}

El yacimiento de Cova Eirós se localiza en la aldea de Cancelo, en el término municipal de Triacastela (Lugo). La entrada a la cavidad se encuentra en la ladera N-NW del Monte Penedo, en la Serra do Ouribio, a unos 780 m.s.n.m. y a 25 m. por encima del arroyo de Bezcos. La cavidad presenta un recorrido de $312 \mathrm{~m}$., una boca de entrada de $2 \mathrm{~m}$. de altura y $3,5 \mathrm{~m}$. de ancho.

Durante la década de los años 80 del pasado siglo, A. Grandal d'Anglade llevó a cabo varias campañas de excavación $(1988,1989$ y 1991) en el interior (sector Pasillo) y el final de la galería principal de la cavidad. El objetivo de esas intervenciones consistía en recuperar restos de úrsidos. Finalizados los trabajos se recuperaron cerca de 4.000 restos óseos pertenecientes a un NMI de 43 Ursus spelaeus (GRANDAL D’ANGLADE 1993).

En el año 1993 se realizó la primera campaña de excavación arqueológica en la cavidad, dentro del "Proxecto Arqueolóxico Val do Sarria-Val do Mao" dirigido por A. A. Rodríguez Casal. Los trabajos consistieron en un sondeo de $1 \mathrm{~m} 2$ en la entrada de la cueva, con el objetivo de valorar su potencial arqueológico. Durante esas labores se identificaron cinco niveles arqueológicos y se recuperaron unos 550 restos líticos adscritos al Paleolítico medio y superior (NogueIRa Ríos 1997). Un año después A. Grandal dirigió una campaña de excavación paleontológica en el sector final de la cavidad.

En 2008 comenzó una nueva etapa de investigación en Cova Eirós, dentro del proyecto "Ocupaciones humanas durante el Pleistoceno de la Cuenca media del Miño" (FÁBregas Valcarce et al. 2009; De LombiDA-Hermida et al. 2020). Los trabajos llevados a cabo en esa nueva etapa, que continúan en la actualidad, arrojaron una secuencia de ocupaciones paleolíticas durante los momentos avanzados del Pleistoceno superior. Hasta el momento se han identificado 6 niveles arqueológicos adscritos al Paleolítico medio (niveles 3, 4 y 5) y al Paleolítico superior (niveles $\mathrm{B}, 1$ y 2), los cuales se han recuperado herramientas líticas y restos faunísticos (DE LOMBERAHERMIDA et al. 2021).

El nivel 5 se caracteriza por limos gruesos con guijarros dispersos de piedra caliza muy angulares. Contiene industria lítica de cronología musteriense. Actualmente en excavación.

En el nivel 4 se identificaron tres facies: 4AI, 4C, 4AS. La 4AI está compuesta de arena muy fina y limos gruesos con concreciones carbonatadas dispersas y algunos cantos rodados de caliza y esquisto. También tiene cantos rodados de caliza muy angulares (espesor: $5 \mathrm{~cm}$ ). Contiene industria Musteriense con productos Levallois y discoidales. La 4C posee limos muy gruesos con concreciones carbonatadas extendidas y cantos rodados de caliza, esquistos y también cantos rodados de caliza muy angulares (espesor: 8-14 cm). Tiene industria Musteriense con producción Levallois. Por último, la 4AS se compone de limos gruesos con algunos cantos rodados dispersos de cuarzo blanco, cuarcita, esquistos y calizas. También contiene cantos rodados muy angulares y cantos rodados de caliza (espesor: 7-12 cm). Contiene una industria Musteriense con Levallois y producción discoidal.

En el nivel 3 se identificaron tres facies con elementos arqueológicos: 3C, 3B y $3 \mathrm{~A}$. En conjunto se puede afirmar que todos contienen industria lítica musteriense. La 3C contiene pequeños guijarros de piedra caliza muy angulares y pequeñas concreciones y laminaciones micríticas de carbonato (espesor: $<12 \mathrm{~cm}$ ). La 3B contiene arena muy fina con cantos rodados muy angulares, dispersos y cantos rodados de piedra caliza (espesor: $8-10 \mathrm{~cm}$ ). Finalmente, la $3 \mathrm{~A}$ se compone por arena muy fina con guijarros dispersos muy angulares y cantos rodados de piedra caliza (espesor: $<25 \mathrm{~cm}$ ).

En el nivel 2 se identificaron dos facies: $2 \mathrm{~A}$ y 2B. La primera contiene una industria lítica $\mathrm{Au}-$ riñaciense. Se caracteriza por arena fina, con pequeños guijarros de piedra caliza, dispersos y con pequeñas concreciones carbonatadas (espesor: $<16$ $\mathrm{cm})$. La 2B no contiene industria lítica diagnóstica, pero presumiblemente se encuadraría en el $\mathrm{Au}$ riñaciense. Su composición se basa en arena muy fina, con cantos rodados muy angulares dispersos, cantos rodados de caliza y pequeñas concreciones carbonatadas (espesor: 6-20 cm).

En el nivel 1 se identificaron 2 facies: $1 \mathrm{~B}$ y 1A. La primera de ellas se compone de arena muy fina, similar al loess, con dispersiones muy angulares y guijarros muy pequeños de piedra caliza. Presenta laminación horizontal (espesor: 5-10 cm). Contiene industria Gravetiense con producción de láminas y elementos de dorso. La 1A se compone de arenas eólicas, con laminación horizontal (espesor: 10-40 cm). No se encontraron artefactos líticos en él. 
También se han identificado los niveles A1 y A2. Se trata de niveles superiores muy extensos y que presentan una pendiente hacia el interior de la cavidad. Se componen de limos difíciles de clasificar que varían hasta arenas muy finas con cantos rodados muy angulares dispersos y cantos rodados de piedra caliza y de cuarcita, transportados antrópicamente. En ellos se encontraron estructuras y cerámica de cronología medieval (CÉSAR et al., 2018).

Durante la Prehistoria Reciente también fue ocupada la cavidad, como demuestra el hallazgo de un vaso con decoración cardial (CuBAs et al. 2020; FÁBREGAS VALCARCE et al. 2019).

Para finalizar, cabe mencionar el hallazgo de evidencias de arte rupestre Paleolítico en el interior de la cavidad (DE LOMBERA-HERMIDA \& FÁbregaS VAlCARCE 2013; GARCÍA-DíEZ et al. 2021). Estas manifestaciones rupestres fueron datadas c. 9.000 cal B.P. (Steelman et al. 2017).

\section{ANTECEDENTES DEL USO DE LOS SIG}

En los últimos años se han llevado a cabo diversos trabajos que empleaban el uso de los SIG, basados en los principios del análisis locacional, y que se han aplicado al estudio del Paleolítico peninsular. Es preciso destacar algunos de ellos en los que se relacionaba el emplazamiento en un determinado sitio con una variable concreta como por ejemplo la vegetación (GARCía MoRENo 2007, 2008b), la insolación (GARCía MORENO 2008a, 2015), la presencia visual (GARCía MORENO 2013c), la movilidad (GARCía Moreno 2013a; Prieto et al. 2016; Ríos-Garaizar \& García MoRENO 2015; SÁNCHEZ et al. 2016) o el clima (BURKe et al. 2014). También se han realizado estudios en los que se incluía más de una variable y que tenían como objetivo definir el patrón de asentamiento y la ocupación de yacimientos paleolíticos localizados en zonas geográficas concretas, como por ejemplo el valle del río Arlanzón (Marcos SÁIz 2006), la cornisa cantábrica (Fernández FernándeZ 2010; García Moreno 2013a; Marín Arroyo 2008, 2009; Ortega MarTÍNEZ \& Ruíz-REDONDO 2017; TuRrero et al. 2013), el valle del río Asón (FANo MARTínEz et al. 2016; García Moreno 2010; García Moreno \& FAno Martínez 2011), el valle del río Nalón (CORChón et al. 2014; García Moreno et al. 2013; García Moreno \& Fano Martínez 2014), la cuenca del río Mundo (GARCÍA Moreno 2014) y el río Sado (Burke et al. 2011).

En el caso concreto del Paleolítico gallego escasean los estudios basados en la aplicación de los SIG. Alguno se centra en el análisis de una o dos variables como la visibilidad y la movilidad en el yacimiento de Valverde, localizado en la Depresión de Monforte de Lemos y adscrito al Paleolítico superior (DE LOMBERA-HERMIDA et al. 2012; RODRÍGUEZ ÁlvAREZ et al. 2008) o por ejemplo el intento de definir las vías de entrada al Noroeste Peninsular (DE LOMBERA-HERMIDA et al. 2011).

Concretamente para la Depresión de Monforte de Lemos se realizó un trabajo en el que se definieron y se analizaron diversas variables que hipo- téticamente estarían marcando el patrón de localización de los yacimientos del Paleolítico ubicados en esa zona. En ese trabajo se analizaron variables como la visibilidad, la altitud, la orientación, la pendiente, la proximidad a los cursos fluviales así como el cálculo de rutas óptimas y su proximidad a los yacimientos arqueológicos (DE LOMBERAHermida et al. 2015). Ese estudio se continuó en la zona de la Serra do Xistral, donde se relacionaron los yacimientos del Paleolítico superior y el Epipaleolítico con la movilidad (DíAz RodríGUEz 2017). En esa área también se analizó la prominencia topográfica de los yacimientos en abrigo, con el objetivo de comprobar si estaban actuando como hitos en el paisaje (Díaz RodríGuez \& CARrero PAzos 2019). También con la aplicación del modelo predictivo al estudio de varias áreas del Paleolítico gallego (DíAz RoDríGUEz 2020).

\section{PLANTEAMIENTO Y OBJETIVOS}

Los diversos restos recuperados durante las intervenciones arqueológicas realizadas en Cova Eirós indican que fue un lugar recurrente de ocupación desde el Paleolítico medio hasta prácticamente la actualidad, siendo utilizado durante la Prehistoria Reciente, la Alta Edad Media y en Época Moderna (DE Lombera-Hermida et al. 2014; RoDRÍGUEZ Álvarez et al. 2011). Los actuales habitantes de Cancelo, entidad poblacional en la que se sitúa la cueva, cuentan que la empleaban como refugio para resguardarse de las inclemencias climáticas cuando se encontraban cuidando del ganado en las inmediaciones. Esto nos lleva a pensar que la localización de la cavidad juega un papel importante en su elección como lugar de refugio o hábitat.

Desde nuestro pensamiento actual no podemos llegar a entender cuáles eran aquellas variables, condiciones o preocupaciones que podían ser importantes para un grupo de individuos del Paleolítico. Sin embargo, podemos intentar aproximarnos a partir del estudio del territorio que se encuentra en el entorno de Cova Eirós. Presuponemos que el aprovisionamiento de agua, materias primas o los recursos cinegéticos deberían de estar disponibles para poder sobrevivir. Por eso, si queremos entender la importancia de Cova Eirós, debemos acudir al estudio de variables como éstas en la superficie próxima a la misma, para poder definir ese patrón de localización. Aunque también debemos destacar que Cova Eirós es una de las pocas cavidades de la zona que ofrece un espacio habitable más o menos amplio y esto también influye en su elección como lugar de ocupación.

Como una de las hipótesis de partida, se planteó que Cova Eirós se encuentre en las proximidades de las rutas naturales que conectan la Meseta con el Noroeste Peninsular. De esta forma se trataría de una localización privilegiada y sería un lugar idóneo para resguardarse, por la amplitud que tiene la entrada de la cavidad, lo que facilitaría la habitabilidad con respecto a otras cuevas que se encuentran en las proximidades, y cuya entrada, zona que emplearían estas comunidades para realizar sus actividades diarias, es más estrecha. 
Además de eso, en las inmediaciones podrían resultar accesibles determinados recursos, como los que se mencionan más arriba, que facilitasen el asentamiento en el lugar, aunque fuese de forma temporal.

\section{METODOLOGÍA}

Mediante los SIG podemos evaluar si este yacimiento se encuentra en las inmediaciones de las rutas de entrada al Noroeste Peninsular y saber si existen, en las proximidades, recursos bióticos o abióticos potencialmente explotables por los pobladores del Paleolítico. No obstante, en primer lugar es necesario definir el nivel de estudio: por un lado necesitamos realizar los análisis de movilidad de Noroeste de la Península Ibérica desde un nivel macroespacial, a gran escala. Además, para determinar la relación del lugar arqueológico con los elementos geográficos que se encuentran en su entorno, estableceremos tres niveles de análisis: un entorno inmediato, un entorno medio y un entorno alejado. De esta forma podemos saber a qué distancia del yacimiento se encuentran los recursos de forma más abundante. Consideramos que la división en tres niveles puede ser de gran relevancia para sociedades de cazadores-recolectores que cuentan con una gran movilidad en comparación con otro tipo de sociedades como son las sedentarias.

Algunas de las variables de primer orden que podrían estar condicionando la localización de los yacimientos paleolíticos han sido definidas previamente (DíAz RodríGUEz 2017: 32-34). En este trabajo vamos a analizar aquellas que pensamos que pueden ser más relevantes para entender este yacimiento y que podrían explicar, de una manera más concreta, dicha localización. Estas variables se dividieron en tres categorías: condicionantes abióticos, condicionantes bióticos y otros condicionantes (Tabla 1).

Tabla 1. Variables de estudio definidas a partir de la bibliografía. Table 1. Study Variables defined from the bibliography.

\begin{tabular}{|c|c|c|c|}
\hline \multicolumn{4}{|c|}{ Análisis locacional } \\
\hline & & Variables & Descripción \\
\hline \multirow{9}{*}{$\begin{array}{l}\text { Factores de } \\
\text { primer } \\
\text { orden }\end{array}$} & \multirow{5}{*}{$\begin{array}{l}\text { Condicionantes } \\
\text { Abióticos }\end{array}$} & Altitud & $\begin{array}{l}\text { Elevación en un punto determinado, en } \\
\text { metros, sobre el nivel del mar. Se calcula a } \\
\text { partir de un MDE. }\end{array}$ \\
\hline & & Pendiente & $\begin{array}{l}\text { Inclinación del terreno en un punto } \\
\text { determinado. Se calcula a partir del MDE. }\end{array}$ \\
\hline & & Hidrología & $\begin{array}{l}\text { Red hidrológica potencial. Calculada a partir } \\
\text { del MDE en función de los puntos que } \\
\text { favorezcan los lugares de acumulación de } \\
\text { agua. }\end{array}$ \\
\hline & & Geología & $\begin{array}{l}\text { Información geológica obtenida a partir de la } \\
\text { Cartografia MAGNA del IGME. }\end{array}$ \\
\hline & & Brañas & $\begin{array}{l}\text { Índice de humedad topográfica, obtenido a } \\
\text { partir del MDE. }\end{array}$ \\
\hline & $\begin{array}{l}\text { Concicionantes } \\
\text { Bióticos }\end{array}$ & MSPLC & $\begin{array}{l}\text { Modelo de Selección de Presas desde un } \\
\text { Lugar Central. Obtenido a partir de datos } \\
\text { etológicos y las pendientes. }\end{array}$ \\
\hline & \multirow{3}{*}{$\begin{array}{c}\text { Otros } \\
\text { condicionantes }\end{array}$} & Visibilidad & $\begin{array}{l}\text { Cálculo del terreno percibido desde un punto. } \\
\text { Obtenido a partir del MDE. }\end{array}$ \\
\hline & & Visibilidad Total & $\begin{array}{l}\text { Número de puntos visibles desde cada uno de } \\
\text { los puntos dados. Calculado a partir del MDE. }\end{array}$ \\
\hline & & Tránsito potencial & $\begin{array}{l}\text { Red potencial de tránsito entre puntos dados. } \\
\text { Se calcula a partir del MDE y teniedo en } \\
\text { cuenta la pendiente y la hidrología. }\end{array}$ \\
\hline
\end{tabular}

Entre los condicionantes abióticos vamos a tener en cuenta la altitud, que podemos definir como la elevación calculada en función de algún punto de referencia, que habitualmente tiende a ser el nivel del mar (si nos referimos a la altitud absoluta) o el fondo de un valle por ejemplo (si hablamos de altitud relativa). Esta variable aparece mencionada en la bibliografía especializada, ya que se considera que los lugares arqueológicos se ubican en puntos elevados con respecto a su terreno circundante, aunque no en lugares excesivamente elevados que pueden dificultar el poblamiento humano (FÁBregas VALCARCE \& DE LOMBERAHermida 2010: 267; DE LOMBERA-HeRMIDA et al. 2015: 285; RAMIL REGO 1989/1990: 194).
Otra de las variables analizada es la pendiente, definida como el grado máximo de variación de la elevación en una determinada posición. Se trata tambien de una variable que ya fue analizada previamente en la bibliografía para otras zonas del Noroeste (DE LOMBERA-HERMIDA et al. 2015: 285).

La hidrología es otra de las variables presentes en el análisis. La relación de los yacimientos paleolíticos con los cursos de auga ha sido defendida en varias ocasiones, a escala regional (FÁbregas VAlCARCE \& DE LOMBERA-HERMIDA 2010: 267; RAMIL Rego 1989: 193; VILLAR QuiNTEIRO 1996). En este caso vamos a utilizar la hidrología potencial, ya que la red hidrográfica es cambiante a lo largo del tiempo y de esta forma vamos 
a aproximarnos lo máximo a la existente utilizando como base el MDE (Modelo Digital de Elevaciones) y teniendo en cuenta aquellos puntos que, topográficamente, son más propensos a actuar como lugares de acumulación de agua.

También tuvimos en cuenta la geología para llevar a cabo los análisis. Para los cazadoresrecolectores del Paleolítico, era importante contar con materias primas, como la cuarcita o el cuarzo en las inmediaciones. Estos recursos podrían obtenerlos a partir de los cursos fluviales, en forma de cantos rodados, o recurriendo a los filones en los que se encuentran esos materiales. La geología ha sido considerada en diversos trabajos previos (FÁbregas VAlCARCE et al. 2010; DE LOMBERAHermida et al. 2012; LóPEZ CORDEIRO 2002, 2015; RAmil Rego 1989; Villar Quinteiro 1996).

La proximidad y el control visual de los yacimientos paleolíticos sobre las zonas de humedal o braña, también ha sido reseñado en varias ocasiones (CRIADO BOADO 1991; DE LOMBERAHermida et al. 2015; López CoRdeiro 2002, 2015). Por eso tendremos en cuenta esta variable, que se podría definir como la acumulación de humedad en un conjunto de puntos.

Por otro lado tenemos los condicionantes bióticos de los que, hasta el momento, solo incluimos una variable que pudimos modelizar. Esto es debido a la dificultad que presenta modelizar otros recursos para períodos tan antiguos, como la vegetación por ejemplo. Esta variable se basa en el cálculo del área de captación de determinados animales que eran cazados por estas comunidades y de los que tenemos vestigios en Cova Eirós. Se trata de un Modelo de Selección de Presas desde un Lugar Central (MSPLC) o Central Place Foraging Prey Choice. Este modelo fue propuesto por M. Cannon (2003) y se basa en la teoría de forrajeo. Posteriormente fue empleado por Marín Arroyo para estudiar los patrones de movilidad y de control del territorio en el Cantábrico oriental. Para ello, el modelo de Cannon se aplicó a la caza de ciervos y cabras como especies más representativas de la dieta magdaleniense (MARín ARroyo 2008, 2009b).

Además de los condicionantes tanto abióticos como bióticos, vamos a tener en cuenta otros condicionantes que pueden estar determinando el patrón de localización. Uno de ellos es la visibilidad o el campo visual, que permite evaluar el conjunto de celdas o puntos que se pueden ver desde un punto dado, en este caso el yacimiento. Esta variable también fue manejada en algunos trabajos previos (FÁBREgAS VALCARCE \& DE LOMBERAHERMidA 2010; DE LOMBERA-HERMIDA et al. 2011, 2015; LÓPEZ CORDEIRO 2002, 2004, 2015; RoDRíGUEz Álvarez et al. 2008).

Otra de las variables que se va a tener en cuenta es la visibilidad total o prominencia visual. Consiste en calcular el número de celdas que son visibles desde cada una de las celdas dentro de una zona acotada (LLOBERA 2003). El proceso de cálculo consiste en obtener la visibilidad desde cada uno

${ }^{(4)} \mathrm{http} / / /$ centrodedescargas.cnig.es/CentroDescargas/. de los puntos que conforman el mapa y luego proceder a sumarlos todos. Se trata de un proceso complejo, computacionalmente hablando, ya que requiere una inversión de tiempo considerable debido a que implica el cálculo de miles de puntos. Creemos que esta variable puede ser interesante, ya que permite saber el número de celdas desde las que es visible aquella en la que se encuentra el yacimiento. En este caso, llevaremos a cabo una simplificación. Por eso en vez de ser visibilidad total, propiamente dicha, se trataría de prominencia visual.

La última de las variables es el cálculo de la intensidad de tránsito potencial, que se va a medir como la relación que existe entre el yacimiento y el desplazamiento por el paisaje colindante. Podemos definirla como la ruta de tránsito generada entre dos puntos, dependiendo de diversos factores y correspondiente al menor coste en energía o tiempo. Las vías naturales de tránsito o los denominados caminos reales han sido tratados previamente en la bibliografía especializada, estableciendo la importancia de estas rutas como una de las variables que marca el patrón de localización de los yacimientos paleolíticos (DÍAz RoDRíGUEZ 2017; FÁBREgas VALCARCE \& DE LOMBERA-HERMIDA 2010: 267; DE LOMBERA-HERMIDA et al. 2015: 289; LÓPEZ CORDEIRO 2015: 301; RAMIL REGO \& RAMIL SONEIRA 1996: 125).

Una vez presentadas y definidas las variables de estudio, pasamos a la elaboración de los análisis. Para ello era necesaria la cartografía base sobre la que se llevó a cabo el estudio. Como el trabajo se realizó en varios niveles de análisis, hemos contado con mapas a distinta resolución. Hemos calculado la intensidad potencial de tránsito a una escala macroespacial, para lo que empleamos el MDE de 200x200 m. de resolución para cada celda o píxel. Para el resto de las variables, el mapa base que se ha utilizado es el MDE de 25x25 m. de resolución. Estos mapas se obtuvieron de la página web del Centro Nacional de Información Geográfica ${ }^{(4)}$.

Contamos también con las coordenadas del yacimiento de Cova Eirós en el sistema de referencia ETRS89 proyección 29N, UTMX: 64686 y UTMY: 473643. Además, para la realización de los análisis, se emplearon los siguientes programas SIG: GRASS GIS 7.0.2 (GRASS DEVELOPMENT TeAm 2016), SAGA GIS 2.2.1 y ArcGIS 10.3 (licencia USC). Para el tratamiento estadístico de los datos se utilizó el programa R 3.3.2 (R Core Team, 2015).

Dado que el Noroeste Peninsular tiene una amplia red fluvial, decidimos emplearla para delimitar nuestra zona de estudio. Pensamos que, a pesar de que las cuencas hidrográficas han ido cambiando, estas modificaciones no han sido de tanta entidad en lo que respecta a los grandes cursos de agua. Las cuencas hidrográficas se definen en relación con un punto específico de un curso de agua (punto de vertido) y abarcan toda la zona que drena ese punto (CONOLLY \& LAKE 2009: 337-339). La metodología usada para el cálculo de las mismas ha sido descrita previamente (DÍAZ RoDRÍ- 
GUEZ 2017: 31). Una vez calculadas, obtuvimos un área acotada que nos sirve como marco para realizar los siguientes análisis (Fig. 2).

La orografía y el paisaje han cambiado a lo largo de los años, de manera que cuanto más retrocedemos en el tiempo, más aumenta la dificultad para reconstruir los territorios del passado.Sin embargo, el período de estudio que nos atañe se centra en los momentos finales del Pleistoceno superior, por lo que consideramos que los cambios que éste pudo sufrir serían menores, sobre todo atendiendo a las principales formaciones orográficas.

Para definir los distintos entornos (inmediato, medio y alejado), utilizamos el cálculo de isócronas. Se trata de un mapa de isolíneas formado por un conjunto de líneas, cada una de las cuales une puntos cuya variable posee el mismo valor. Las mismas no pueden cruzarse, ya que significaría que en un mismo punto estarían presentes dos valores (Olaya 2014: 693). Las isócronas son una medida de tiempo, que nos permite saber cuánto nos llevaría desplazarnos desde un punto de origen (en este caso el yacimiento de Cova Eirós) a cualquiera de las celdas del entorno. El cálculo de desplazamiento se hizo teniendo en cuenta un coste de desplazamiento anisotrópico, que se caracteriza porque la resistencia que presenta al desplazarse depende tanto de la dirección del viaje como de cualquier otro atributo direccional de cada una de las celdas del mapa.

El entorno inmediato se definió teniendo en cuenta un desplazamiento de una distancia máxima de 1 hora. El entorno medio se estableció a una distancia máxima de 4 horas, constituyendo, en el borde de esta isócrona, el desplazamiento máximo al que podían llegar los habitantes de Cova Eirós, dándoles tiempo a regresar al yacimiento con la luz del día. Por último, el entorno alejado se estableció en el límite de la isócrona de 8 horas, entendiendo que ésta sería la media anual aproximada de duración de la luz solar (FÁBREGAS VALCARCE \& RODRÍGUEZ Rellán 2017: 75) (Fig. 3).

Dentro de cada uno de los entornos decidimos comparar el resultado de los análisis de las variables para Cova Eirós, con 999 puntos creados y distribuidos aleatoriamente. Creemos que estadísticamente suponen un número suficiente para mostrar la tendencia general de cada una de las variables en cada uno de los entornos y así poder hacer una comparación con el yacimiento.

\subsection{ALTITUD}

El cálculo de la altitud se realizó teniendo en cuenta la altitud absoluta de Cova Eirós obtenida del MDE. Para saber si esta variable es relevante, la comparamos con 999 puntos aleatorios en el entorno inmediato, medio y alejado. Además analizamos tanto los puntos aleatorios, como la totalidad de las celdas que conforman cada una de las zonas de estudio con el yacimiento. De esta forma podemos ver si los valores de los puntos aleatorios son representativos del resto del terreno y si coinciden o no con Cova Eirós (Fig. 4A).

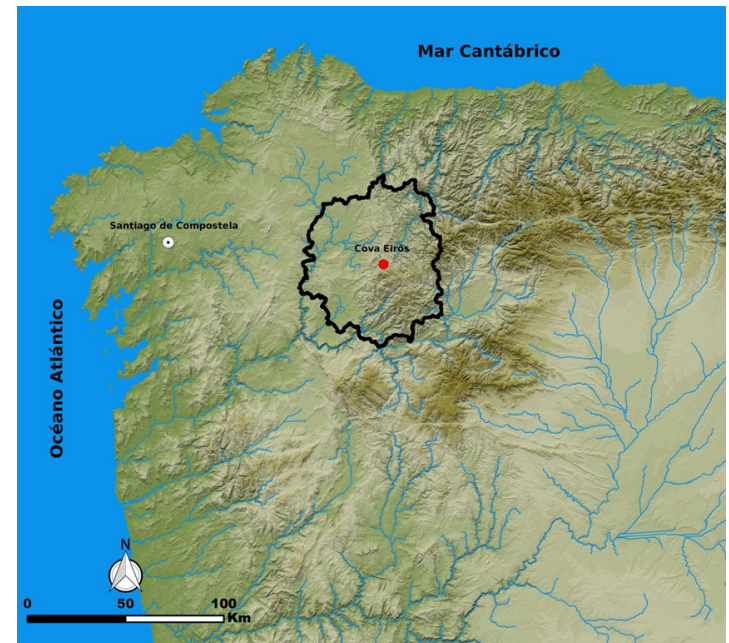

Fig. 2. Zona acotada a partir de las cuencas hidrográficas (línea negra). Localización de Cova Eirós (punto rojo).

Fig. 2. Area limits as defined from the watershed (black line). Location of Cova Eirós (red dot).

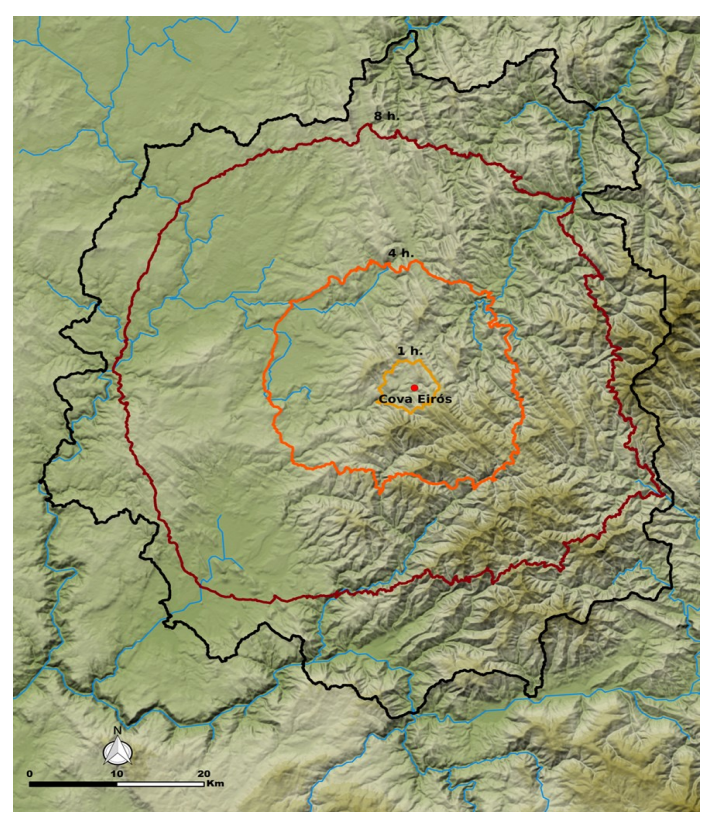

Fig. 3. Límite del entorno inmediato (1 hora), medio (4 horas) y alejado (8 horas).

Fig. 3. Limit of the near environment (1 hour), middle (4 hours) and remote ( 8 hours)

\subsection{PENDIENTE}

La pendiente se estableció clasificando en cuatro grupos los valores obtenidos a partir del mapa de pendientes generado en función del MDE. De esta forma, basándonos en trabajos previos (GARCía MoReno 2010) hemos establecido una zona llana (pendientes entre 0 y $5 \%$ ), terreno ondulado (entre 5 y $15 \%$ ), terreno abrupto (entre 15 y $30 \%$ ) y roquedo (más del $30 \%$ ).

Una vez establecidas esas categorías, se comparó la pendiente de los puntos aleatorios con el yacimiento de Cova Eirós en cada uno de los 
entornos de estudio. Además también se comparó la pendiente de los puntos aleatorios con la pendiente de todas las celdas del mapa en cada uno de los entornos (Fig. 4B).

\subsection{HIDROLOGÍA POTENCIAL}

La relación del yacimiento de Cova Eirós con los cursos de agua se estableció realizando un cálculo de desplazamiento en términos temporales. O sea, evaluando el tiempo necesario para llegar desde el yacimiento, o desde cualquier celda del mapa, a la fuente acuífera más próxima.

Para eso, se calcularon los principales cursos de agua. En primer lugar, se eliminaron aquellas celdas con valores extraños o negativos del MDE, ya que podrían dar problemas a la hora de realizar el cálculo. Estos errores pueden ser debidos a la propia configuración del MDE o a la interpolación del mismo.

Una vez que el mapa base fue corregido, creamos una capa de drenaje (flow direction),
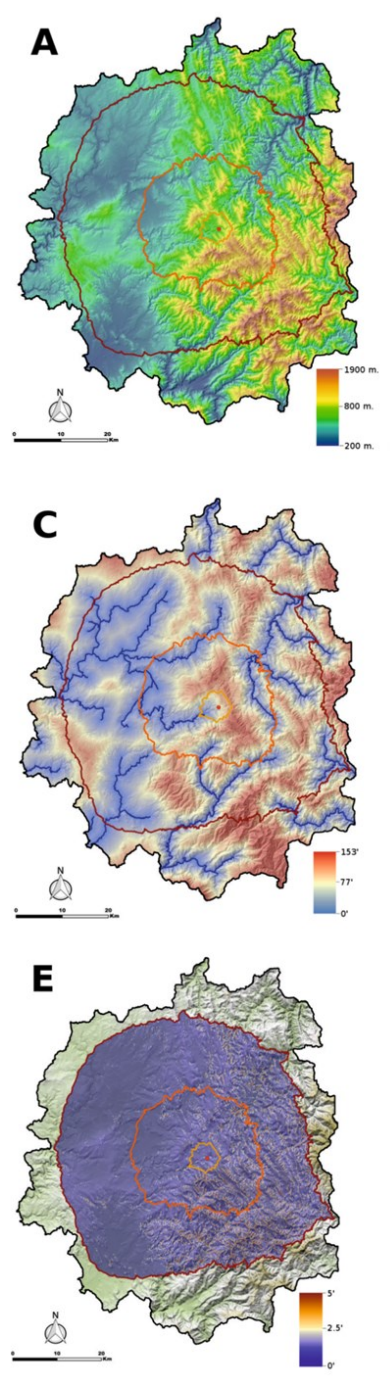

que indica la dirección que seguiría una masa de agua artificial que discurriese por el MDE. Una vez obtenidos esos datos, calculamos la dirección que sigue este flujo, permitiendo al mismo tiempo ver la acumulación de varios flujos, o sea, se trata de identificar cuantas celdas del mapa drenan hacia otra celda (flow accumulation).

El mapa de drenaje obtenido nos muestra los cursos de agua de la zona de estudio. Cada uno tiene un valor ordenado de forma jerárquica y este valor se incrementa siempre que dos cursos, que tengan el mismo orden, se junten. Dado que los cursos actuales de drenaje no se corresponden con los que podía haber en períodos más antiguos, decidimos seleccionar aquellos cuyo valor numérico fuese alto, por encima del tercer cuartil, ya que se trata de cursos de agua con cierta entidad de los que es más fácil que tuviesen una mayor pervivencia en el tiempo. Los ríos así obtenidos fueron descompuestos en puntos para calcular el coste desde ellos al resto de celdas de los entornos de estudio (Fig. 4C).
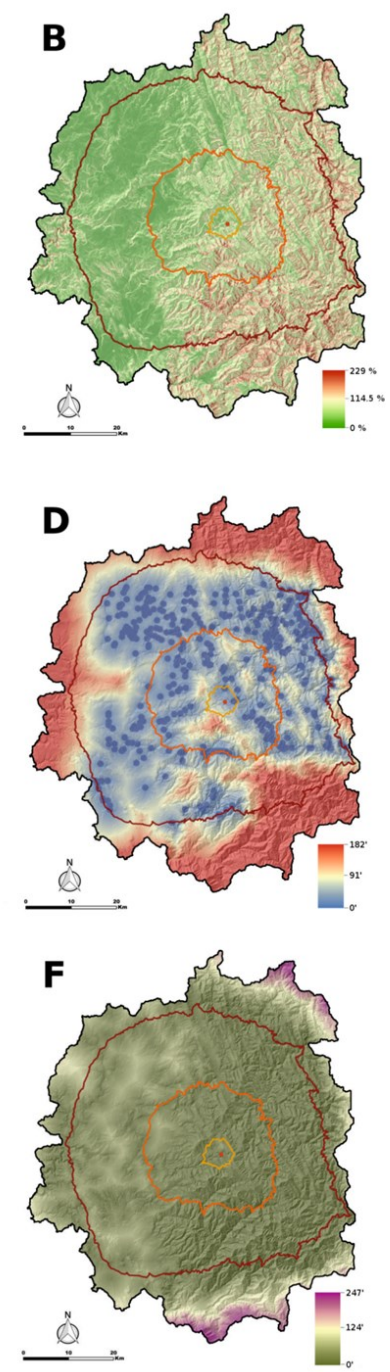

Fig. 4. Mapas en los que se muestran algunos de los análisis realizados. A) Altitud, B) Pendiente, C) Coste recursos hídricos, D) Coste recursos litológicos, E) Coste a zonas de humedal y F) Coste a zonas de captación de cápridos.

Fig. 4. Maps showing some of the analyses performed. A) Altitude, B) Slope, C) Cost to water resources, D) Cost to lithological resources, E) Cost to wetland areas and F) Cost to goat catchment areas. 


\subsection{RECURSOS LITOLÓGICOS POTENCIALES}

Consideramos que la geología también jugaba un papel importante para estas sociedades, ya que explotaban los recursos líticos que se encontraban en algunas formaciones geológicas para emplearlos como herramientas. En los conjuntos de Cova Eirós, el material lítico hegemónico es el cuarzo, seguido de la cuarcita y, para los niveles del Paleolítico superior, el cristal de roca y el sílex (este último con porcentajes menores al 4\% (DE LOMBERA-Hermida et al. 2014). Además, el análisis de la corticalidad de los artefactos líticos indica que la mayoría procede de la recolección en depósitos secundarios (terrazas, coluvios), aunque el cristal de roca y el sílex podrían adquirirse en formaciones primarias o subprimarias. Por eso decidimos identificar aquellas formaciones susceptibles de ser explotadas a partir del mapa geológico MTN a escala 1:50000 (Hojas 98, 99, 124, 125, 156 y 157) tales como las cuarcitas de Cándana, armoricanas o los depósitos cuaternarios (entre otros). Una vez identificadas fueron convertidas a puntos y se calculó la proximidad, en coste de desplazamiento en tiempo, desde éstas hasta el yacimiento arqueológico y también hasta los puntos aleatorios dentro de cada una de las zonas de estudio (Fig. 4D).

\subsection{ZONAS DE HUMEDAL}

Para el cálculo de las brañas o zonas de humedal, empleamos una metodología que ha sido desarrollada en trabajos previos (BöHNER et al. 2001; Carrero Pazos 2017: 151). Consiste en crear un mapa ráster de humedales, donde las celdas representan una tendencia de escorrentía, de tal forma que se considera que un área que posea una mayor humedad será más propensa a saturarse y a conformar un humedal.

Se trata de una variable que ha cambiado a lo largo del tiempo, y sobre todo teniendo en cuenta el clima del Noroeste Peninsular. Sin embargo, pensamos que es preciso hacer una aproximación de este estilo para poder estudiar, de la mejor forma posible, el paisaje del pasado.

En este caso se utilizó el módulo de SAGA GIS, Topographic Wetness Index (TWI), que nos permite calcular la acumulación de flujo en función de la pendiente del terreno. El resultado que nos da este análisis es un mapa en el que se muestra un índice de humedad para cada una de las celdas del mapa. Posteriormente decidimos emplear, como representativas, aquellas celdas que tenían un valor superior al tercer cuartil, de tal forma que sería en dichas celdas en las que se formarían las brañas o zonas de humedal. Una vez obtenidas, se calculó la proximidad al yacimiento y a los puntos aleatorios de cada uno de los entornos, como se hizo con anterioridad para otras variables (Fig. 4E).

\subsection{MSPLC}

Las variables que tratamos anteriormente, las definimos como condicionantes abióticos, ya que se trata de elementos o factores ambientales que no poseen vida propia ni se relacionan con ella. Sin embargo, los elementos bióticos también jugarían un papel importante en la vida de los habitantes de Cova Eirós. De esta forma, tuvimos en cuenta una metodología basada en el denominado Modelo de Selección de Presas desde un Lugar Central o Central Place Foraging Prey Choice Model (MSPLC), que se basa en el cálculo de los tiempos de desplazamiento en un determinado territorio y se aplica a la caza de ciervos y cabras, como especies más representativas de la dieta magdaleniense (CANNON 2003; MARÍn ARroyo 2008) y cuyos restos han sido identificados en Cova Eirós (VALVERdE TEJEDOR 2019).

De esta forma se aplicó la Teoría del Forrajeo Óptimo para estimar el límite máximo de productividad asociado a cada especie. Así, teniendo en cuenta un punto de origen, la distancia máxima de caza para distintos animales deberá estar en relación con la energía asociada a cada uno de ellos y a los costes de su procesado y transporte (CANNON 2003).

Como resultado, se estableció un tiempo de viaje máximo, para que la captura del ciervo suponga la mayor productividad posible, de 2,15 horas. El mismo cálculo, aplicado a la caza de la cabra, dio como resultado un valor de 1,2 horas. Por otro lado, dentro de esa área de captación es preciso establecer la superficie en la que determinada especie puede vivir atendiendo a las características ecológicas de la misma. A falta de datos ambientales más precisos y correspondientes a esa época, se consideró que la pendiente sería el factor más determinante a la hora de establecer las áreas potencialmente explotables por animales de hábitats llanos (como el ciervo) y aquellos de roquedo (como la cabra), ya que la altitud o la exposición solar presentan un rango de validez para cada especie poco limitante. En función de la pendiente, se estableció que las especies asociadas a hábitat de montaña estarían situadas por encima de $\operatorname{los} 30^{\circ}$ de pendiente, y aquellas asociadas a zonas llanas se encontrarían por debajo de los $30^{\circ}$ de pendiente (Marín Arroyo 2008: 34-35).

Para evaluar las zonas de captación, tanto de ciervo como de cabra, se utilizó el yacimiento de Cova Eirós como punto de partida. Se tuvieron en cuenta las isócronas de 2,15 y 1,2 horas y dentro de ellas las celdas con pendiente superior e inferior a $30^{\circ}$. Posteriormente se calculó la proximidad de coste en tiempo de desplazamiento desde esas celdas al yacimiento. Se hizo el mismo proceso para los puntos aleatorios de cada uno de los entornos, calculando las isócronas desde ellos y comparando los resultados de esos puntos con los del yacimiento, tanto para las cabras (Fig. 4F) como para los ciervos (Fig. 5A).

\subsection{VISIBILIDAD}

En cuanto al cálculo de la visibilidad, tuvimos en cuenta la elaboración de un análisis simple, de tipo binario. Distinguiendo aquellas celdas visibles desde el yacimiento (con valor 1 ) de aquellas no visibles (con valor 0). Para comparar ese dato en cada uno de los entornos de estudio, 
calculamos también la visibilidad desde los puntos aleatorios. Como zona límite de visibilidad tuvimos en cuenta la zona de estudio previamente acotada (Fig. 5B).

A la hora de obtener la visibilidad es importante tener en cuenta la altitud del observador y del terreno. Es preciso invertirlas para obtener un cálculo más realista (CONOLLY \& LAKE 2009: 300). Es decir, si nuestro yacimiento es el punto A, y cualquier celda del terreno es el punto $\mathrm{B}$, lo ideal sería que la altura del punto A fuese 0 y la del punto B se correspondiese con la altura del observador. En este caso optamos por la altura media de un Homo sapiens, que establecemos en 1,75 m. para el observador y $0 \mathrm{~m}$. para el punto de inicio.

\subsection{PROMINENCIA VISUAL}

La prominencia visual es una simplificación de la visibilidad total, que al mismo tiempo puede definirse como la visibilidad inherente a todas las localizaciones en un paisaje (LlOBERA et al. 2010).
Consiste en calcular la visibilidad desde todas las celdas del mapa, para posteriormente contar el número de celdas de todo el campo visual y registrar el número de celdas que son visibles desde allí (CONOLly \& LAKE 2009: 298). El resultado es un mapa en el que tienen valores más altos aquellas zonas que son más prominentes visualmente.

En nuestro caso, decidimos aproximarnos al cálculo de la visibilidad total mediante una fórmula muestral. Se trata de una metodología que ha sido empleada en trabajos anteriores (CARRERO PAzos 2017: 158). Este método consiste en ubicar múltiples puntos distribuidos a lo largo del área de estudio y calcular la visibilidad acumulada desde cada uno de ellos. Para eso se generó una malla de puntos regular por toda el área de estudio, espaciados a una distancia de 500 metros entre cada uno de ellos.

Posteriormente se calculó la prominencia en cada una de las celdas en las que se ubican, tanto el yacimiento, como los puntos aleatorios de cada uno de los entornos de estudio (Fig. 5C).
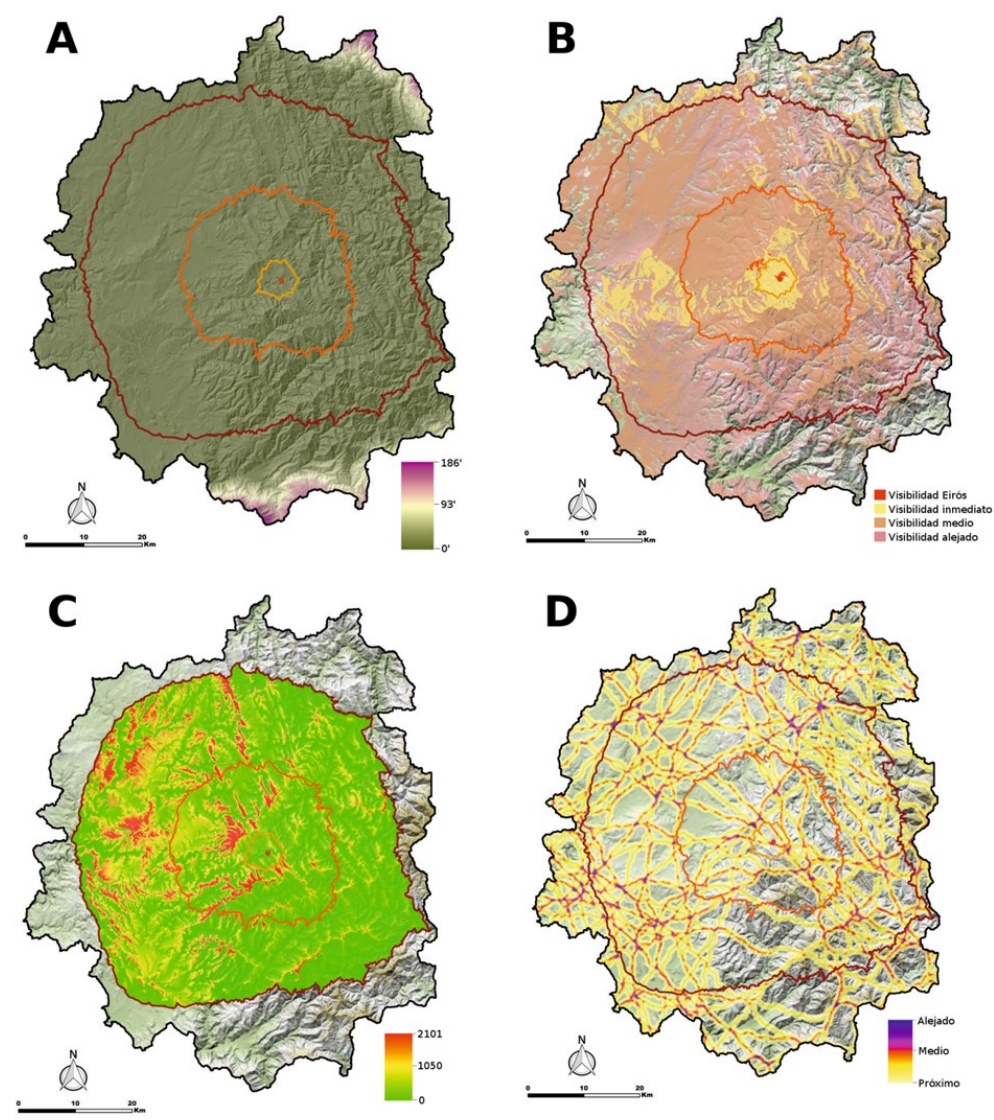

Fig. 5. Mapas en los que se muestran algunos de los análisis realizados. A) Coste a zonas de captación de ciervos, B) Visibilidad, C) Prominencia visual, D) Intensidad de tránsito potencial.

Fig. 5. Maps that show some of the analyses performed. A) Cost to deer catchment areas, B) Visibility, C) Visual prominence, D) Potential transit intensity.

\subsection{INTENSIDAD DE TRÁNSITO POTENCIAL}

El último de los análisis realizados fue el cálculo de la intensidad de tránsito potencial. La metodología utilizada ha sido descrita en un trabajo anterior (DíAz RoDRÍGUEz 2017) para el que se empleó el programa GRASS GIS. Sin embargo, en esta ocasión se trataba de un cálculo realizado a una escala más amplia. Por eso el mapa se amplió a 200×200 m. de resolución para cada celda del 
mapa, como ya se comentó. De esta forma es más sencillo de obtener, ya que con una resolución muy alta sería necesario recurrir a la supercomputación para el cálculo de estos datos.

La zona de estudio para este análisis fue definida a partir de las cuencas hidrográficas, como se explicó anteriormente. Una vez establecida, se generaron puntos separados a una distancia de $10.000 \mathrm{~m}$. entre sí y localizados a lo largo del contorno de la zona de estudio. En total se generaron 208 puntos. Desde cada uno de esos puntos se calculó la ruta de menor coste a los 207 puntos restantes. Para calcular ese coste tuvimos en cuenta, como superficie de fricción, la acumulación hídrica y la pendiente. Calculadas ambas empleando el MDT como mapa base.

En el siguiente paso se calculó el coste que existe desde cada uno de los puntos al resto. Este proceso se repitió para cada uno de los puntos. Una vez obtenido cada uno de los mapas se generaron las rutas de menor coste de tránsito. Tras realizar este proceso se obtuvieron 43.056 polilíneas o rutas que se unieron en un único archivo, a partir del cual se creó un kernel que refleja la intensidad de tránsito potencial, y que indica aquellas áreas que pudieron actuar como nodos en la red de tránsito (Fig. 5D).

Por último, se obtuvo un mapa de coste acumulado que nos permitió calcular la distancia, en minutos, desde las rutas al yacimiento y a los puntos aleatorios de cada uno de los entornos.

\section{RESULTADOS}

Una vez analizadas las variables decidimos tratarlas y representarlas estadísticamente, empleando a tal fin los boxplots o gráficos de cajas y también los gráficos de barras.

\subsection{ALTITUD}

Para la primera de ellas, la altitud, podemos observar que la mediana de los puntos generados de forma aleatoria se localiza por encima del yacimiento de Cova Eirós, en el entorno inmediato. Los puntos situados en el entorno medio presentan una tendencia similar a la del yacimiento arqueológico. Sin embargo, la mediana de aquellos situados en el entorno alejado marca una altitud inferior a la de Cova Eirós, exceptuando algún outlier que se dispara a altitudes próximas a los 1.500 m.s.n.m. Esto es debido a que al ampliar el terreno, sobre todo hacia el oeste, se encuentran amplias zonas bajas en las que se reduce significativamente la altitud (Fig. 6A).

Para saber si el análisis de los puntos aleatorios era representativo de los entornos de estudio, decidimos calcular la altitud de cada una de las celdas de nuestro MDE, agrupándolas en rangos y otorgándole a cada uno, un porcentaje del total de las celdas contenidas en ese rango y comparamos el porcentaje de celdas del terreno con el porcentaje de puntos aleatorios. Lo que pudimos observar es que, a grandes rasgos, el porcentaje de celdas de
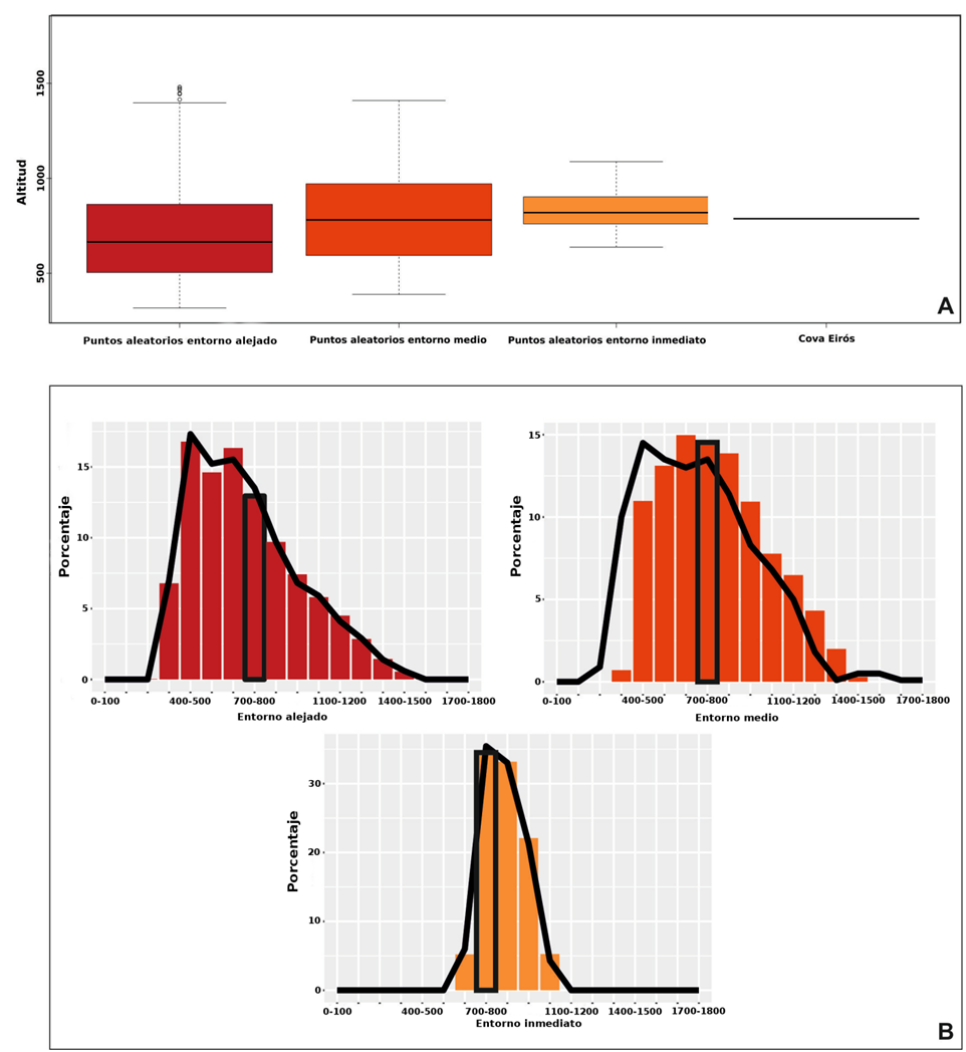

Fig. 6. A) Diagramas de caja comparativos de la altitud en los tres entornos y en Cova Eirós. B) Rangos de altitud de todas las celdas del terreno (barras), puntos aleatorios (línea negra) y Cova Eirós (columna destacada en negro).

Fig. 6. A) Comparative boxplot of the altitude in the three environments and in Cova Eirós. B) Altitude range of all terrain cells grouped into four categories (bars, random points (black line) and Cova Eirós (column highlighted in black). 
todo el mapa se corresponde con el porcentaje de puntos aleatorios en el terreno inmediato y donde, además, Cova Eirós se localiza en el rango más representado (los 700-800 m.s.n.m.). La tendencia general del entorno medio es algo diferente del anterior, ya que el rango de altitudes más representadas, para los puntos aleatorios, es el de $400-500$ y para el terreno es el de 600-700 m.s.n.m. En este entorno, Cova Eirós se localiza en el segundo rango más representado, en lo que al porcentaje del terreno se refiere y que coincide con los puntos aleatorios. Por último, la comparación de los puntos aleatorios con el terreno, en el entorno alejado, coincide prácticamente en el mismo rango, cobrando más importancia la localización altitudinal de Cova Eirós, que se ubica en un rango que en ningún caso es el más representado ya que ni tan siquiera llega al 15\% del total (Fig. 6B).

\subsection{PENDIENTE}

En lo que respecta a la pendiente, es importante señalar que Cova Eirós se encuentra en una ladera, com una fuerte pendiente, en torno a un $115 \%$ de inclinación. Cuando comparamos la pendiente con los puntos aleatorios de todos los entornos, se encuentra ampliamente por encima de la totalidad de los puntos del entorno inmediato y del medio (Fig. 7A), y solo existe un outlier que sobrepasa el $120 \%$ en el entorno alejado.

Siguiendo la división del terreno, en función del porcentaje de inclinación de la pendiente que se mencionó en el apartado metodológico, comparamos el porcentaje de todas las celdas de los distintos entornos con el de puntos aleatorios para ver si eran representativos o no. Dando como resultado una coincidencia casi idéntica en el entorno inmediato, siendo el terreno ondulado el más representado, seguido de cerca por el abrupto, luego la zona llana y por último el de roquedo. Sin embargo, es en este último donde se encuentra el yacimiento de Cova Eirós. Con respecto a los entornos medio y alejado, ocurre que el roquedo es lo menos representado en el terreno, pero el más recurrente en cuanto a los puntos aleatorios (Fig. 7B).
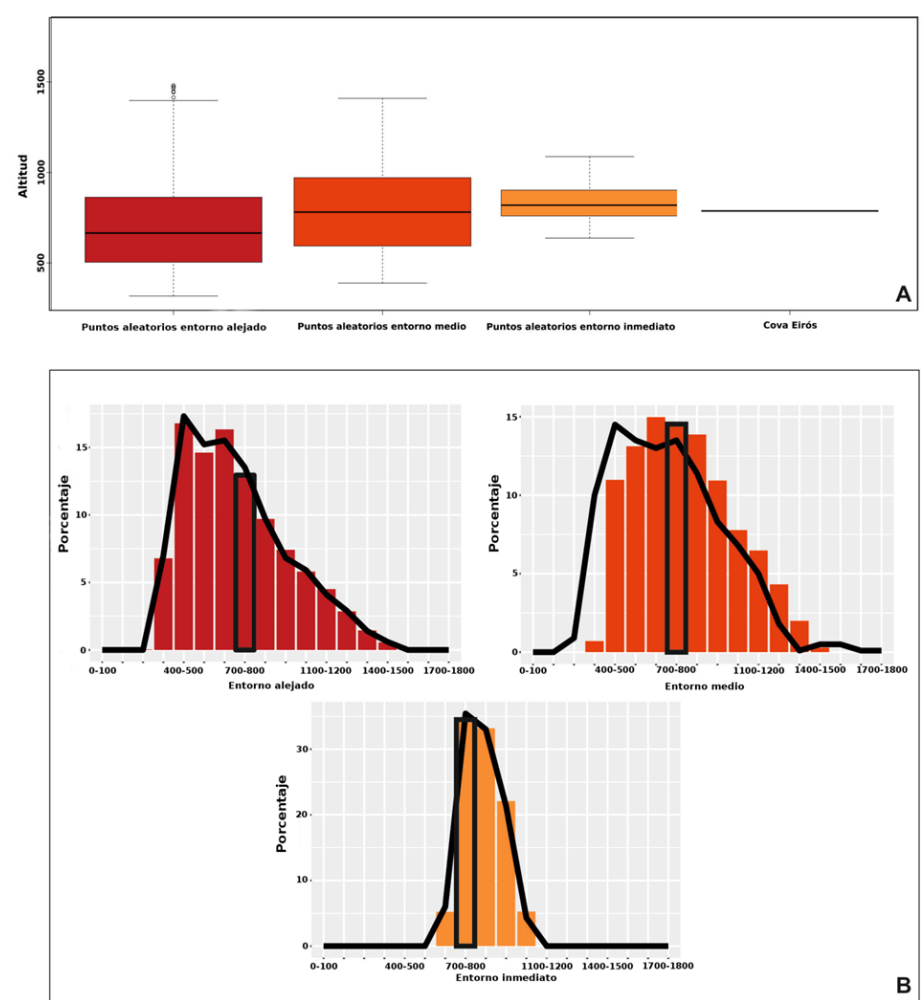

Fig. 7. A) Diagramas de caja comparativos de la altitud en los tres entornos y en Cova Eirós. B) Rangos de altitud de todas las celdas del terreno (barras), puntos aleatorios (línea negra) y Cova Eirós (columna destacada en negro).

Fig. 7. A) Comparative boxplot of the altitude in the three environments and in Cova Eirós. B) Altitude range of all terrain cells grouped into four categories (bars, random points (black line) and Cova Eirós (column highlighted in black).

\subsection{HIDROLOGÍA POTENCIAL}

La cuantificación, con fines comparativos de la hidrología potencial se llevó a cabo obteniendo el coste de desplazamiento en tiempo desde las celdas en las que se sitúa el recurso hídrico hasta el yacimiento de Cova Eirós y hasta los puntos aleatorios de cada uno de los entornos. De este modo obtuvimos que el cauce de mayor entidad más próximo del yacimiento se localiza a unos 60 minutos. Este valor está situado ligeramente por debajo de la mediana de los puntos aleatorios del entorno inmediato. El valor de la mediana sube ligeramente en el entorno medio y es prácticamente similar al de Cova Eirós. Por último, los puntos aleatorios del entorno alejado reflejan una variabi- 
lidad mayor con respecto a los otros dos entornos, quizás por la mayor amplitud de terreno, y en los que la mediana muestra un dato inferior a los 50 minutos (Fig. 8A).

\subsection{RECURSOS LITOLÓGICOS POTENCIALES}

Con respecto a la litología, la metodología empleada es similar a la de la variable anterior, teniendo en cuenta el coste de desplazamiento en minutos. Si bien, el abastecimiento de cuarzos y cuarcitas puede solventarse en el regato inmediato de Bezcas, cabe destacar que, en este caso, el yacimiento se encuentra a unos 24 minutos del afloramiento primario susceptible de ser explotado más próximo (filones de Monte de Albela). Lo que resulta interesante, ya que este valor es inferior a la mediana de todos los entornos. Los puntos de abastecimiento para las cuarcitas armoricanas o los sílex de origen calcáreo se encuentran a mayor distancia. En lo tocante a los entornos medio y alejado, hay algunos outliers que se encuentran a distancias de casi 120 minutos, lo que contrasta con el resto de puntos, que están más próximos (Fig. 8B).
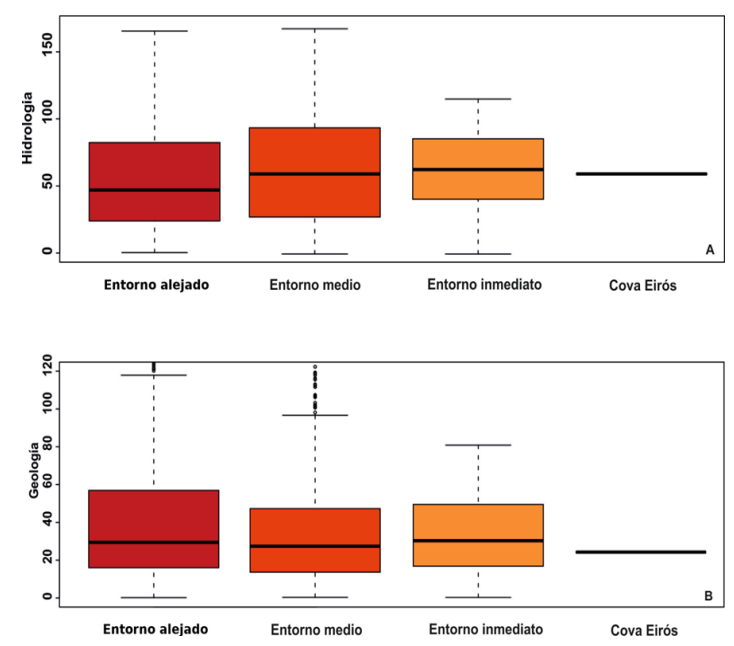

Fig. 8. A) Diagramas de caja comparativos del coste desde la hidrología potencial a los puntos aleatorios de los tres entornos y a Cova Eirós. B) Diagramas de caja comparativos del coste desde la geología potencial a los puntos aleatorios de los tres entornos y a Cova Eirós.

Fig. 8. A) Comparative boxplot of the cost from the potential geology to the random points of the three environments and Cova Eirós. B) Comparative boxplot of the cost from the potential geology to the random points of the three environments and Cova Eirós.

\subsection{ZONAS DE HUMEDAL}

La proximidad de las zonas de humedal al yacimiento de Cova Eirós nos dio un valor de 2 minutos. Comparamos este valor con los datos de los puntos aleatorios del entorno inmediato, medio y alejado. Además hemos dividido los datos en rangos de 2 minutos de 0 a 10, lo que nos permitió observar que la mayoría del porcentaje de estos puntos se encuentra a menos de 2 minutos en todos los entornos. Posteriormente decidimos comparar la media de los 999 puntos de cada uno de los entornos con Cova Eirós. Esto nos permitió apreciar que estos puntos aleatorios están por debajo de los 0,5 minutos de proximidad de las zonas de humedal. Ante la similitud que presenta esta variable en todos los puntos aleatorios de los tres entornos, pensamos que no parece relevante a la hora de marcar el patrón de emplazamiento. A pesar de que los 2 minutos del yacimiento se sitúen por encima en los tres casos, ya que también están indicando una localización muy próxima a la cavidad (Fig. 9).
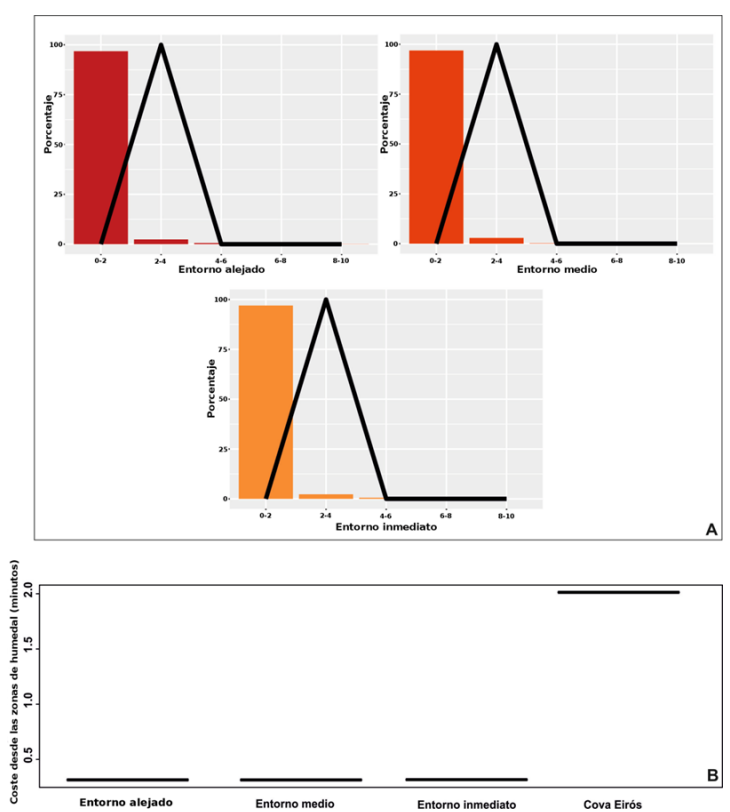

Fig. 9. A) Gráfico de barras que indica el porcentaje de coste desde las zonas de humedal (barras) y el de Cova Eirós (línea negra). B) Media del coste de los 999 puntos aleatorios en cada entorno y el dato de Cova Eirós.

Fig. 9. A) Barplot displaying the percentage of cost from the wetland areas (bars) and Cova Eirós (black line). B) Average cost of 999 random points in each environment and Cova Eirós.

\subsection{MSPLC}

El MSPLC se calculó para dos especies representativas del Pleistoceno superior como son la cabra y el ciervo. Para cuantificar las zonas de captación potencial de ambas especies, decidimos hacerlo teniendo en cuenta el coste de desplazamiento desde aquellas celdas del mapa, en las que se podrían cazar, hasta el yacimiento y también hasta los puntos aleatorios para cada uno de los entornos. Para cuantificarlo y compararlo se establecieron rangos de 15 minutos desde 0 a 75 minutos.

Para las cabras se observa que en los entornos inmediato y medio aproximadamente el $80 \%$ de los puntos aleatorios se encuentran en los primeros 15 minutos de desplazamiento. Para el entorno alejado este valor baja del $75 \%$. Esto podría ser debido a lo ya observado en otras variables, que al ampliar el territorio de estudio la problemática varía en función de la propia geografía del terreno (Fig. 10A). Este dato se aprecia también al calcular la media de los puntos aleatorios de cada entorno y al compararla con Cova Eirós. En el entorno inmediato los puntos aleatorios se encuentran a 8 minutos de media, en el medio se sitúa ligeramente por encima 
de los 6 minutos mientras en el alejado sube hasta los 12. Por el contrario, el yacimiento se localiza por encima de una celda en la que podrían habitar las cabras. Sin embargo, de encontrarse humanos en el yacimiento, los animales se alejarían hasta estar a salvo (Fig. 10B).

Con respecto a los ciervos, su hábitat es más amplio y ocupa una mayor superficie del terreno que el de la cabra. Al comparar los rangos en los distintos entornos, apreciamos que en el inmediato se encuentra representado prácticamente el $90 \%$ de los puntos aleatorios en los primeros 15 minutos.
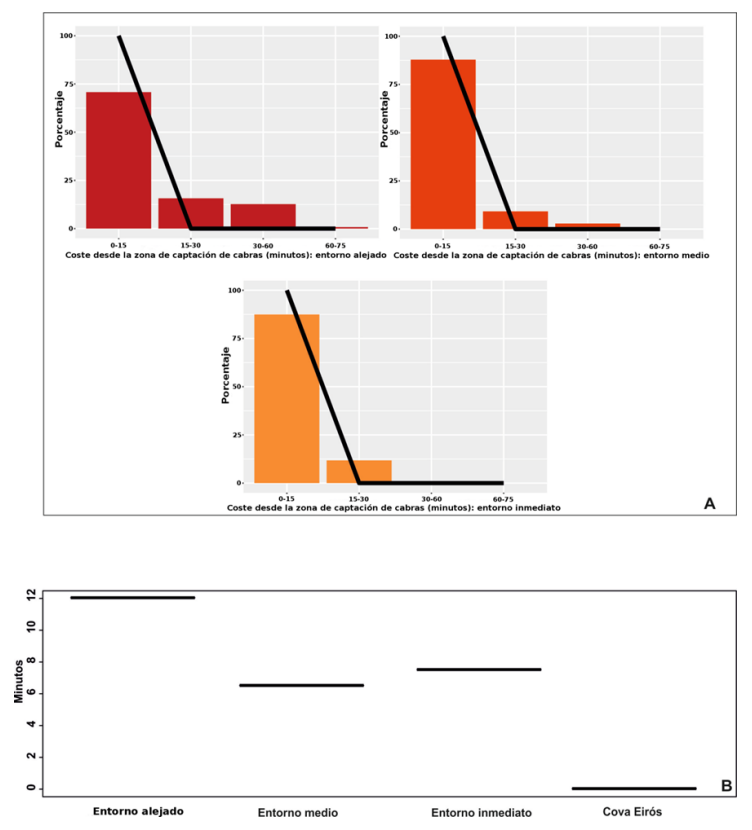

Fig. 10. A) Gráfico de barras que indica el porcentaje del coste desde la zona de captación de cabras (barras) y el de Cova Eirós (línea negra). B) Media del coste de los 999 puntos aleatorios en cada entorno y el dato de Cova Eirós.

Fig. 10. A) Barplot displaying the percentage of cost from the goat catchment area (bars) and Cova Eirós (black line). B) Average cost of 999 random points in each environment and Cova Eirós.

\subsection{VISIBILIDAD}

Para realizar el cálculo de la visibilidad decidimos calcular la superficie de terreno visible desde el yacimiento (en hectáreas) y compararla con la media de los puntos aleatorios localizados en cada uno de los entornos. A pesar de que Cova Eirós se encuentra en una ladera, como ya se comentó con anterioridad, y esto limita su visibilidad hacia el noroeste, su dominio sobre el valle inmediato permite que desde ese punto se observen 500 hectáreas de terreno. Este dato contrasta con la media de los puntos aleatorios en el entorno inmediato ya que el valor de éstos es de unas 36 ha. Según ampliamos el terreno, esta cifra aumenta. En el entorno medio asciende hasta las 180 ha. y en el alejado sube a las 300 ha. Sin embargo, son datos que quedan muy por debajo de la visibilidad obtenida desde el yacimiento (Fig. 12A).
Mientras que en el medio y el alejado el 100\% de los puntos se encuentran dentro de esos 15 minutos (Fig. 11A).

Vuelve a ser representativo este dato si lo complementamos con las medias de los entornos. De esta forma apreciamos que en Cova Eirós la zona más próxima de captación potencial de ciervo se sitúa cerca de los 2 minutos. En el inmediato la media se encuentra en los 6,5 minutos, sin embargo en el medio y en el alejado es inferior a 1 minuto, por lo que esos puntos aleatorios están casi encima de estas celdas (Fig. 11B).
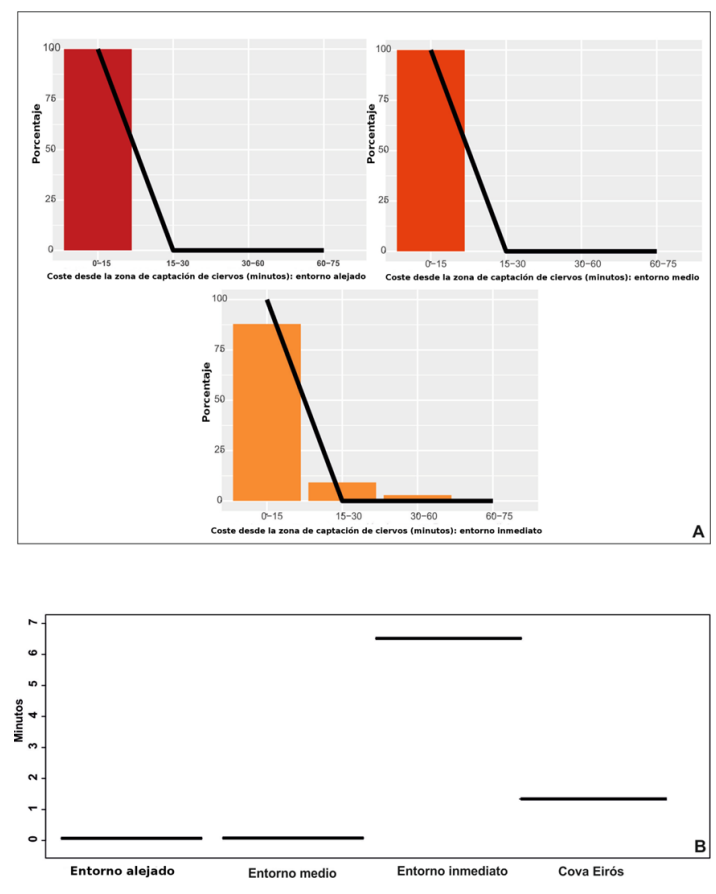

Fig. 11. A) Gráfico de barras que indica el porcentaje de coste desde la zona de captación de ciervos (barras) y el de Cova Eirós (línea negra). B) Media del coste de los 999 puntos aleatorios en cada entorno y el dato de Cova Eirós.

Fig. 11. A) Boxplot displaying the percentage of cost from the deer catchment area (bars) and Cova Eirós (black line). B) Average cost of 999 random points in each environment and Cova Eirós.

\subsection{PROMINENCIA VISUAL}

Para la cuantificación de la prominencia visual, se tuvo en cuenta el número de celdas visibles desde cada una de las celdas en las que se ubica Cova Eirós y los puntos aleatorios de cada uno de los entornos. Cuanto más alto es el valor en una celda más prominente visualmente será dicho. Por tanto, destaca por su visibilización en el terreno. En este caso el valor que dio para Cova Eirós en cada uno de los entornos es distinto, ya que cabe recordar que para el análisis se tienen en cuenta puntos repartidos uniformemente por la zona de estudio en cuestión, en este caso por cada uno de los tres niveles de entorno. Para el primero de ellos, el entorno inmediato, el valor del yacimiento se localiza ligeramente por debajo de la mediana de los puntos aleatorios. Lo mismo ocurre en el medio, aunque en éste, la mediana de esos puntos aleatorios, tiende a dispararse, con outliers 
que llegan hasta un valor de 350. Ampliando la zona, esta tendencia continúa y los puntos que, en el medio eran considerados outliers, en el alejado entran dentro de la zona de control del diagrama de caja, o sea dentro del valor máximo (Fig. 12B).

\section{9. ÍNDICE DEL TRÁNSITO POTENCIAL}

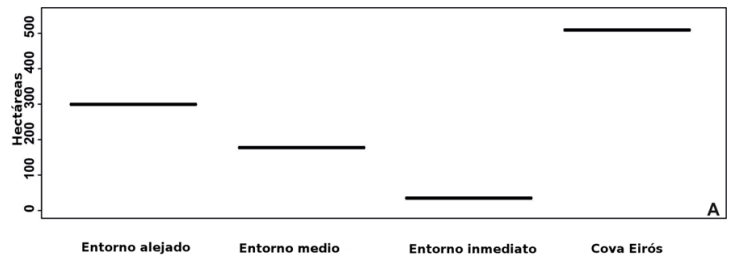

El cálculo de rutas óptimas se representó en un gráfico de barras, en el que se indica el rango del mismo modo que el del MSPLC. En el entorno inmediato, el $87 \%$ de los puntos aleatorios se sitúan a menos de 15 minutos de las rutas de menor coste. Este valor desciende en el entorno medio hasta el $79 \%$ y vuelve a subir en el alejado hacia el 84\% (Fig. 13A). Con respecto a la media, Cova Eirós se localiza a menos de 2 minutos de la ruta más próxima. Los puntos aleatorios del entorno inmediato están en torno a 7 minutos de media, mientras que en el entorno medio este valor sube casi a los 10 minutos. Por último, en el entorno alejado los puntos se localizan en valores ligeramente superiores a los 8 minutos (Fig. 13B).

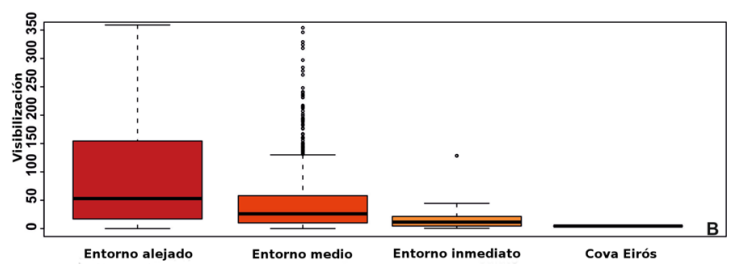

Fig. 12. A) Media de la superficie visible (hectáreas) en cada uno de los entornos y en Cova Eirós. B) Diagramas de caja de la prominencia visual en cada uno de los entornos y en Cova Eirós.

Fig. 12. A) Average of the visible surface (hectares) in each of the environments and in Cova Eirós. B) Boxplot of visual prominence in each of the environments and in Cova Eirós.
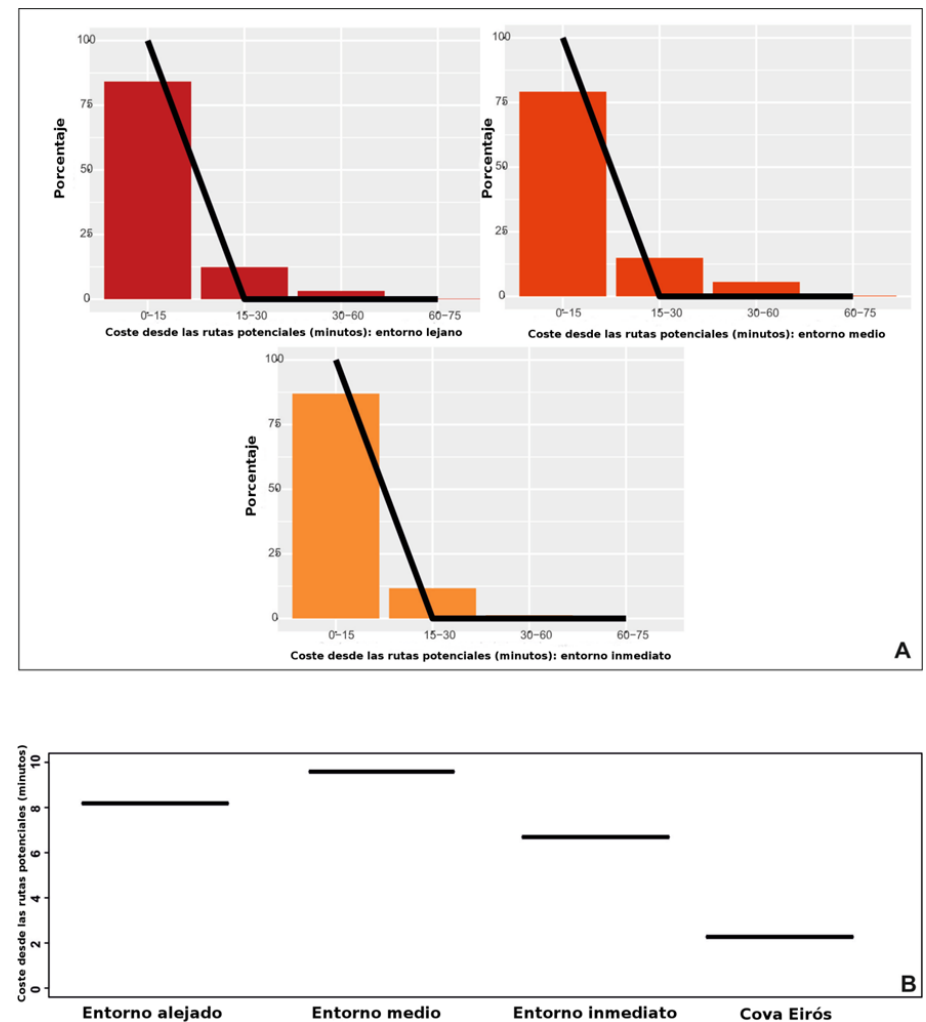

Fig. 13. A) Gráfico de barras que indica el porcentaje del coste desde las rutas de tránsito potencial (barras) y el de Cova Eirós (línea negra). B) Media del coste de los 999 puntos aleatorios en cada entorno y el dato de Cova Eirós.

Fig. 13. A) Boxplot displaying the percentage of cost from the least cost path (bars) and Cova Eirós (black line). B) Average cost of 999 random points in each environment and Cova Eirós.

\section{DISCUSIÓN Y CONCLUSIONES}

La elaboración de este estudio nos permitió realizar una aproximación al conocimiento del entorno del yacimiento de Cova Eirós, sobre todo de cara a intentar definir el patrón de localización de este lugar arqueológico. La división en tres entornos nos mostró que cuanto mayor es el territorio estudiado, más varía éste, por lo que es más difícil detectar tendencias. En nuestro caso, ese cambio puede observarse entre el entorno inmediato y el medio. Este último presenta mayores similitudes con el alejado, mientras que el inmediato, por norma general, presenta características más 
similares al lugar en el que se encuentra el yacimiento paleolítico.

Ante la falta de yacimientos arqueológicos afines a Cova Eirós, que nos permitiesen hacer un análisis estadístico completo, decidimos emplear 999 puntos aleatorios en cada entorno. Estos puntos, al compararlos con una localización escogida, en base a criterios humanos, deberían diferenciarse del yacimiento por su aleatoriedad. Una vez realizado el análisis, pensamos que es una muestra lo suficientemente representativa ya que, al compararla con el resto de celdas del mapa en variables como la altitud o la pendiente, estos puntos representan acertadamente la tendencia de las celdas.

En cuanto a las variables que definimos al comienzo del estudio y que podrían estar marcando el patrón de ocupación del yacimiento, pudimos apreciar que entre ellas la altitud no destaca excesivamente, aunque podría ser importante al localizarse por encima de la media en entornos amplios $\mathrm{y}$, en los momentos más rigurosos de la última glaciación, próxima a cotas vinculadas con depósitos glaciares o periglaciares (VIANA-SOTO \& Pérez-Alberti 2019). Por otro lado, la pendiente sí que nos parece que juega un papel importante. Se trata de una variable que está directamente relacionada con otras, como la captación potencial de las cabras o que afecta a la visibilidad y que definitivamente destaca en este estudio.

La hidrología potencial no da indicios de jugar un papel destacado, localizándose a 1 hora de proximidad al cauce de mayor entidad, aunque la tendencia que existiría en los entornos más próximos al yacimiento, reduce este tiempo a partir de las 4 horas, cuando el terreno es más llano. De nuevo debemos tener en cuenta las fluctuaciones climáticas ya que durante los periodos más templados y húmedos es muy probable que los riachuelos inmediatos tuvieran caudales más estables, tal y como indican las aproximaciones paleoclimáticas (REY-RodRíguez et al. 2016). En lo tocante a la litología, se trata de una variable que cobra cierta importancia. Los muestreos hechos en los depósitos secundarios del entorno inmediato muestran que éstos pueden satisfacer gran parte de la demanda en cuarzos y cuarcitas, que son los materiales predominantes. En cambio, para otras variedades de cuarzo, el cristal de roca y el sílex, tendrían que recurrir a los afloramientos del entorno medio o lejano.

La distancia a la que se encuentran las zonas de humedal no es lo suficientemente contrastada como para poder tenerla en cuenta. Al igual que la variable anterior, sería importante depurarla más, introduciendo nuevos estudios derivados de esta variable como, por ejemplo, el control visual de estas zonas de humedal desde el yacimiento, frente al control desde otros puntos aleatorios y comparando los datos obtenidos. Se trata de una hipótesis que ha sido mencionada con anterioridad pero que aún no ha sido comprobada y que deberemos abordar en futuros estudios.

La zona de captación de las cabras, calculada en coste, demostró una cierta relevancia, por lo que podría tratarse de otra variable a tener en cu- enta. Nos queda pendiente poder cuantificar, por ejemplo en forma de superficie, el terreno disponible para ser explotado en el entorno del yacimiento y compararlo con el de los puntos aleatorios. A priori, si estuviésemos ante una variable importante, además de la proximidad, debería influir también la superficie próxima explotable. En el caso del ciervo, a pesar de que en el entorno inmediato el valor de Cova Eirós destaca por su proximidad con respecto a la media de los puntos aleatorios, no es tan diagnóstico como el anterior. Sería interesante buscar otros factores que ayuden a depurar esta variable aparte de la pendiente y del límite en tiempo de la zona de captación, como por ejemplo la visualización desde el yacimiento teniendo en cuenta la altura de los animales. O incluso haciendo a la inversa, ya que si los animales detectan movimiento y observan a los humanos, esas celdas en las que se encontrarían potencialmente los ciervos, dejarían de ser seguras para ellos por lo que habría que descartarlas.

Otra de las variables que destacó en cuanto a su valor por encima de los puntos aleatorios fue la visibilidad. La gran superficie visible desde Cova Eirós, convierte el yacimiento en un punto estratégico y de control. Su orientación hacia el valle le permite poseer ese control visual que probablemente resultó de gran interés para los pobladores de esta cavidad. Sin embargo, ocurre lo contrario con la prominencia visual. Esta variable es inferior a la mediana de todos los entornos, por lo que no destaca por su visualización desde otras zonas del mapa. Lo que también puede resultar positivo, ya que se trata de un lugar resguardado y difícil de detectar.

Por último, la intensidad de tránsito potencial está muy vinculada al yacimiento: a menos de 2 minutos se encuentra una ruta de entrada al Noroeste Peninsular. Su proximidad resulta, cuando menos, destacada debido a que se trata de una localización con una cierta altitud y una fuerte pendiente. Algo que en el entorno alejado se reduce y que debería llevar a que la media de la proximidad a las rutas se redujese por tratarse de zonas llanas que facilitan el paso. La proximidad a estas rutas confirma su importancia como enclave estratégico de paso, sobre todo porque en las inmediaciones también se observa un nodo o cruce de caminos natural. Cabe recordar su proximidad con el Camino de Santiago, vía histórica de peregrinaje y conexión con la Meseta occidental.

El estudio de las variables mencionadas nos lleva a pensar en Cova Eirós como un punto neurálgico, destacado y estratégico. Lo que se confirma atendiendo a esta última variable, el tránsito, unido al fuerte control visual que existe sobre las inmediaciones. Pero sin olvidar que se trata de una zona resguardada y difícilmente perceptible desde el terreno circundante, marcada por una fuerte pendiente que también dificulta el acceso. Además de que posee recursos alimenticios fácilmente explotables en las inmediaciones, tales como la caza de cabras y ciervos. Del mismo modo también se encuentran en las proximidades las zonas de captación de materias primas. 


\section{AGRADECIMIENTOS}

Las intervenciones arqueológicas en Cova Eirós han sido financiadas por la Consellería de Cultura de la Xunta de Galicia (2015-2021) y se enmarcan en el proyecto de investigación "Dinámicas poblacionales y tecnológicas durante el Pleistoceno final-Holoceno de las Sierras orientales del Noroeste ibérico" (Proyectos de $\mathrm{I}+\mathrm{D}+\mathrm{i}$ del Ministerio de Ciencia, PID2019-107480GBI00). La investigación de X.P.Rodríguez-Álvarez se enmarca en el grupo de investigación SGR2017 -1040 (Generalitat de Catalunya). El Institut Català de Paleoecologia Humana i Evolució Social (IPHES-CERCA) recibe apoyo financiero del Ministerio de Ciencia e Innovación de España a través del programa de Unidades de Excelencia "María de Maeztu" (CEX2019-000945-M).

\section{BIBLIOGRAFIA}

Böhner, J., Köthe, R., Conrad, O., Gross, J., Ringeler, A., \& Selige, T. 2001. Soil regionalisation by means of terrain analysis and process parameterisation. En: E. Micheli, F. Nachtergaele \& L. Montanarella. (Eds.), Soil Classification: 213 -222 .

Burke, A., Levavasseur, G., James, P. M. A., GuiduccI, D., Izquierdo, M. A., Bourgeon, L., Kageyama, M., RAMSTEIN, G. \& VRAC, M. 2014. Exploring the impact of climate variability during the Last Glacial Maximum on the pattern of human occupation of Iberia. Journal of Human Evolution, 73: 35-46. 10.1016/j.jhevol.2014.06.003

Burke, A., Meignen, L., Bisson, M., Pimentel, N., Henriques, V., Andrade, C., da Conceição Freitas, M., Kageyama, M., Fletche, R., W., Parslow, C. \& GuiducCI, D. 2011. The Palaeolithic occupation of southern Alentejo: the Sado River Drainage Survey. Trabajos de Prehistoria, 68(1): 25-49. 10.3989/tp.2011.11057

CANNON, M. D. 2003. A model of central place forager prey choice and an application to faunal remains from the Mimbres Valley, New Mexico. Journal of Anthropological Archaeology, 22: 1-25. 10.1016/ S0278-4165(03)00002-3

Carrero Pazos, M. 2017. El Fenómeno tumular y megalítico en Galicia. Aportaciones desde los Sistemas de Información Geográfica y la estadística espacial para el estudio de los patrones de localización. Tesis Doctoral, Universidade de Santiago de Compostela.

César, V., M. De Lombera Hermida, A. Fábregas ValCARCE, R., \& Rodríguez-Álvarez, X.-P. (2018). Estudio de la cerámica medieval de Cova Eirós (Triacastela, Lugo). Cuadernos de Estudios Gallegos, 65 (131): 73-105. https://doi.org/10.3989/ ceg.2018.131.03

Conolly, J., \& LaKe, M. 2009. Sistemas de Información Geográfica aplicados a la arqueología. Barcelona, Bellaterra arqueología.

Corchón, M. S., Fano, M. Á., Gárate, D., García moreno, A., Rivero, O., \& Ortega, P. 2014. La ocupación del valle del Nalón durante el período 13,2-11,5 ky BP: el contexto magdaleniense de La Peña de Candamo (Asturias). En: M. S. Corchón \& M. Menéndez (Eds.), Cien años de arte rupuestre paleolítico, Salamanca: 221-244.
Criado Boado, F., Bonilla Rodríguez, A., Cerqueiro Landín, D., Díaz VÁzouez, M., González MÉndez, M., INFANTE Roura, F., MÉNDEZ Fernández, F., Penedo Romero, R., Rodríguez Puentes, E. \& Vaquero Lastres, J. 1991. Arqueología del paisaje, el área Bocelo-Furelos entre los tiempos paleolíticos y medievales (campañas de 1987,1988 y 1989), Santiago de Compostela (A Coruña), Dirección Xeral do Patrimonio Cultural.

Cubas, M., Lucquin, A., Robson, H. K., Colonese, A. C. Arias, P., Aubry, B., Billard, C., Jan, D., Diniz $\mathrm{M}$, FERnandes, R., Fábregas Valcarce, R, Germain-Vallée, C., Juhel, L., de LomberaHermida, A., Marcigny, C., Mazet, S. Marchand, G., Neves, C., Ontañón-Peredo, R. Rodríguez-Álvarez, X. P., Simões, T., Zilhão, J. \& Craig, O. E. 2020. Latitudinal gradient in dairy production with the introduction of farming in Atlantic Europe Nature Communications 2020 11:1, 11(1): 1-9. https://doi.org/10.1038/s41467020-15907-4.

de Lombera-Hermida, A., Díaz Rodríguez, M., Pérez Alberti, A., Ameijenda Iglesias, A., Rodríguez Álvarez, X. P., \& Fábregas Valcarce, R. 2015. Evolución de los patrones de asentamiento de los yacimientos paleolíticos de la Depresión de Monforte de Lemos (Lugo, Galicia). Sémata: Ciencias Sociais e Humanidades, 27: 267-297.

de Lombera-Hermida, A., \& Fábregas Valcarce, R 2013. Cova Eirós: primeras evidencias de arte rupestre paleolítico en el noroeste peninsular. Santiago de Compostela (A Coruña): Andavira.

de Lombera-Hermida, A., Rodríguez Álvarez, X.-P., AMEIJENDA-IGLESIAS, A., DÍAZ-Rodríguez, M Rey-Rodríguez, I., Valverde TeJedor, I., PÉrezAlberti, A., Cunha, P., Bal García, H., Aldea Moreira, X., Lorenzo Salgueiro, C., Mosouera CAstro, T., FÁbregas-VAlcARCE, R., 2021. Between two worlds: Cova Eirós and the MiddleUpper Palaeolithic transition in NW Iberia. Comp tes Rendus Pale vol, 20 (42): 859-886. 10.5852/crpalevol2021v20a37.

de lombera-Hermida, A., Rodríguez Álvarez, X. P., FÁBregas Valcarce, R., \& LaZuÉN FernándeZ, T. 2011. The Paleolithic Settlement of the Monforte Basin (Lugo, Galicia). En: A. de Lombera Hermida \& R. Fábregas Valcarce (Eds.), To the West of Spanish Cantabria. The Paleolithic Settlement ofGalicia, Oxford, BAR International Series 2283: 93-110.

De Lombera-Hermida, A., Rodríguez Álvarez, X. P. \& FÁbregas VALCARCE, R. 2014. El yacimiento arqueo-paleontológico de Cova Eirós (Triacastela, Lugo). En: E. Carbonell, J. Bermúdez de Castro \& J. L. Arsuaga (Eds.), Los cazadores recolectores del Pleistoceno y del Holoceno en Iberia y el Estrecho de Gibraltar. Estado actual del conocimiento del registro arqueológico, Universidad de Burgos: $18-25$.

de Lombera-Hermida, A., Rodríguez Álvarez, X. P., Rabuñal Gayo, X., AmeiJenda Iglesias, A. Martínez Gómez, F., SoAres Remeseiro, M., Pérez Alberti, A. \& Fábregas Valcarce, R 2012. El yacimiento de Valverde (Monforte de Lemos, Lugo, Galicia, España) y las primeras evidencias de poblamiento en el Pleniglacial del NO de la Península Ibérica. Espacio, Tiempo y Forma. Serie I, Prehisoria y Arqueología, 5: 363 382. 10.5944/etf

de Lombera-Hermida, A., Rodríguez Álvarez, X.-P., Valverde Tejedor, I., \& FÁbregas Valcarce, R. 
2020. Entre dos mares. Los últimos neandertales y los primeros artistas del yacimiento de Cova Eirós (Lugo, Galicia). In A. Carretero Pérez \& C. Papí Rodes (Eds.), Actualidad de la Investigación Arqueológica en España II (2019-2020). Conferencias impartidas en el Museo Arqueológico Nacional, Ministerio de Cultura y Deporte: 185-201.

DíAz RodríGuez, M. 2017. Patrones de asentamiento en el Paleolítico superior y el Epipaleolítico en el Noroeste Peninsular. Cálculo de rutas óptimas y movilidad. En: A. Vázquez Martínez, R. Cordeiro Macenlle, M. Carrero Pazos, M. Díaz Rodríguez, A. A. Rodríguez Nóvoa, \& B. Vilas Estévez (Eds.), (Re)escribindo a Historia. Achegas dos novos investigadores en Arqueoloxía e Ciencias da Antigüidade, Santiago de Compostela (A Coruña): $25-46$.

DíAz RoDríGuez, M. 2020. Tecnologías Geoespaciales aplicadas al estudio del Paleolítico en el Noroeste Peninsular. Tesis Doctoral, Universidade de Santiago de Compostela.

Díaz Rodríguez, M., \& Carrero Pazos, M. 2019. La Prominencia Topográfica de los yacimientos en abrigo en las Sierras Septentrionales (Lugo, Galicia). Una aproximación de estudio con SIG. En: Los tiempos cambian, de la piedra al teclado. $X$ Jornadas de Jóvenes en Investigación Arqueológica, Burgos:363-373.

Fábregas Valcarce, R., Alonso Fernández, S., AmeiJenda Iglesias, A., Grandal D'Anglade, A., LazuÉn Fernández, T., DE Lombera Hermida, A., Pérez Alberti, A., Pérez Rama, M., Rodríguez Álvarez, X. P., Rodríguez Rellán, C., Serna González, M. R., Terradillos Bernal, M. \& VAquero Rodríguez, M. 2009. Novos resultados das intervencións arqueolóxicas no sur lucense. Os xacementos paleolíticos da Depresión de Monforte (Monforte de Lemos), Cova Eirós (Triacastela) e Valdavara (Becerreá). Gallaecia, 28: 9-32.

Fábregas Valcarce, R., Alonso Fernández, S., AmeiJENDA Iglesias, A., Grandal D'Anglade, A., Lazuén Fernández, T., de Lombera Hermida, A., Pérez Alberti, A., Pérez Rama, M., Rodríguez Álvarez, X. P., Serna González, M. R. \& VAQuero Rodríguez, M. 2010. Completando o mapa. Novas datacións absolutas para o Paleolítico e Mesolítico do interior galego. Gallaecia, 29: 528.

Fábregas Valcarce, R., Faustino Carvalho, A., De Lombera-Hermida, A., Cubas, M., Lucquin, A., Edward Craig, O., \& Rodríguez-Álvarez, X. P. 2019. Vaso con decoración cardial de Cova Eirós (Triacastela, Lugo). Trabajos de Prehistoria, 76 (1): 147-160. https://doi.org/10.3989/ tp.2019.12231

Fábregas Valcarce, R. \& de Lombera Hermida, A. 2010. El paleolítico superior en Galicia a la luz de las últimas investigaciones. En: X. Mangado (Ed.), El Paleolítico superior peninsular. Novedades del siglo XXI. Homenaje al Profesor Javier Fortea. Monografíes SERP, 8, Barcelona, Universitat de Barcelona: 255-270.

FÁbregas Valcarce, R. \& RodríGuez Rellán, C. 2017. Rasgos básicos de la extracción de variscita en Palazuelo de las Cuevas (Zamora). Zephyrus, 79: 63-79 10.14201/zephyrus2017796379

Fano Martínez, M. Á., García Moreno, A., Chauvin, A., Clemente-Conte, I., Costamagno, S., Elorrieta-Baigorri, I., Pascual, N. E. \& TARriño, A. 2016. Contribution of landscape analysis to the characterisation of Palaeolithic sites: A case study from El Horno Cave (northern Spain). Quaternary International $\quad 412 . \quad 82-98$. 10.1016 j.quaint.2015.10.105

Fernández Fernández, J. 2010. Una aportación desde la arqueología del paisaje al conocimiento del primer poblamiento humano del Valle del Trubia. Estudio geoarqueológico y análisis SIG del territorio, Oviedo, Universidad de Oviedo.

García-Diez, M., Viñas, R., DE Lombera-Hermida, A., Rubio, A., Rodríguez-Álvarez, X. P., Ochoa, B., \& FÁbregas-Valcarce, R. 2021. Cova Eirós (Galicia, Spain): The 'Finisterre' of Paleolithic cave art. Journal of Archaeological Science: Reports, 35, 102756. https://doi.org/10.1016/ j.jasrep.2020.102756

García Moreno, A. 2007. La evolución del paisaje en la transición al holoceno. desarrollo de un modelo predictivo de vegetación en el valle del Asón (cantabria). Trabajos de Prehistoria, 64(2): 55-71.

García Moreno, A. 2008A. Insolación y hábitat Paleolítico en el Valle del Asón (Cantabria, España). Cuaternario y Geomorfología, 22(3-4): 93-105.

García Moreno, A. 2008B. Predictive Models and the Evolution of Tree Vegetation during the Final Pleistocene-Holocene Transition: A Case Study from the Asón River Valley (Cantabria, Spain). En: A. Posluschny, K. Lambers, I. Herzog (Eds.), CAA2007. Layers of Perception. Proceedings of the 35th International Conference on Computer Applications and Quantitative Methods in Archaeology, Berlin, Germany, April 2-6, 2007 (Kolloquien zur Vor- und Frühgeschichte, Vol. 10), Bonn: 392398).

García Moreno, A. 2010. Patrones de asentamiento y ocupación del territorio en el Cantábrico Oriental a final del Pleistoceno: Una aproximación mediante SIG. Tesis Doctoral Universidad de Cantabria.

García Moreno, A. 2013A. GIS-based methodology for Palaeolithic site location preferences analysis. A case study from Late Palaeolithic Cantabria (Northern Iberian Peninsula). Journal of Archaeological Science, 40(1): 217-226. 10.1016 j.jas.2012.08.023

García Moreno, A. 2013B. Mobility Models and Archaeological Evidence: Fitting Data into Theory. En: P. R. Preston (Ed.), Mobility, Transition and Change in Prehistory and Classical Antiquity. Proceedings of the Graduate Archaeology Organisation Conference on the Fourth and Fifth of April 2008 at Hertford College, Oxford, UK: 83-94.

García Moreno, A. 2013C. To see or to be seen... is that the question? An evaluation of palaeolithic sites' visual presence and their role in social organization. Journal of Anthropological Archaeology, 32 (4): 647-658. 10.1016/j.jaa.2013.03.003

García Moreno, A. 2014. El poblamiento paleolítico de la cuenca del río Mundo (Albacete). Archivo de Prehistoria Levantina, XXX:1-16.

García Moreno, A. 2015. The Incidence of Potential Insolation on Settlement Dynamics and Site Location Preferences: A Case Study From the Cantabrian Late Palaeolithic. Journal of Archaeological Science: Reports, 3: 90-99. 10.1016/j.jasrep.2015.05.020

García Moreno, A., \& Fano Martínez, M. Á. 2011. Los sitios paleolíticos en su paisaje: la Cueva del Horno en el contexto de la cuenca del río Asón (Cantabria). Zephyrus, LXVII: 15-26.

García Moreno, A., Fano Martínez, M. Á. \& Gárate, D. 2013. Integrating spatial analyses into foraging 
societies land use strategies. A case study from the Nalón river basin (Asturias, North of Spain). En: G., Earl, T. Sly, A. Chrysanthi, P. Murrieta-Flores, C. Papadopoulos, I. Romanowska \& D. Wheatley (Eds), Archaeology in the Digital Era. Papers from the 40th Annual Conference of Computer Applications and Quantitative Methods in Archaeology (CAA), Southampton, 26-29 March 2012, Amsterdam, Amsterdam University Press: 670-677.

García Moreno, A., \& Fano Martínez, M. Á. 2014. Paleolithic sites beyond the archaeological deposits. En: A. García, J. García, A. Maximiano, \& J. Ríos-Garaizar (Eds.), Debating Spatial Archaeology: Proceedings of the International Workshop on Landscape and Spatial Analysis in Archaeology (Santander, June 8th-9th, 2012), Santander: 231241.

Grandal D'Anglade, A. 1993. El oso de las cavernas en Galicia: el yacimiento de Cova Eirós, Sada (A Coruña), Ediciós do Castro.

Grass Development Team. 2016. Geographic Resources Analysis Support System (GRASS) Software. Recuperado de http:/grass.osgeo.org/ programming7/

Llobera, M. 2003. Extending GIS-based visual analysis: the concept of visualscapes. International Journal of Geographical Information Science, 17(1): 2548. $10.1080 / 713811741$

Llobera, M., Wheatley, D., Steele, J., Cox, S., \& ParCHMENT, O. 2010. Calculating the inherent visual structure of a landscape (inherent viewshed) using high-throughput computing. Beyond the artefact: Digital Interpretation of the Past: Proceedings of CAA2004, Prato, 13-17 April 2004, (Tomlin 1990): 146-151. 10.1007/s10816-012-9139-2

López Cordeiro, M. DEL M. 2002. Ocupación de espacios graníticos en la Sierra de Xistral: el área arqueológica de Nordés (Valadouro, Lugo). Gallaecia, 21: 61-86.

LóPez Cordeiro, M. DEL M. 2004. Resultados obtenidos en la construcción de una «Nueva Arqueología» del paleolítico gallego. Cuadernos de Estudios Gallegos, LI(117): 133-151.

López CordeIro, M. DEL M. 2015. Industrias Líticas y Ocupación del espacio en el Paleolítico de Galicia. Tesis Doctoral, Universidade de Santiago de Compostela.

Marcos Sáiz, F. J. 2006. La Sierra de Atapuerca y el Valle del Arlanzón. Patrones de asentamiento prehistóricos. Burgos

Marín Arroyo, A. B. 2008. Patrones de movilidad y control del territorio en el Cantábrico oriental durante el Tardiglaciar. Trabajos De Prehistoria, 65: 2945 .

Marín Arroyo, A. B. 2009. The use of optimal foraging theory to estimate Late Glacial site catchment areas from a central place: The case of eastern Cantabria, Spain. Journal of Anthropological Archaeology, 28(1): 27-36. 10.1016/ j.jaa.2008.11.001

NogueIRa Ríos, S. 1997. Unha primeira aproximación o estudio dos materiais líticos de Cova Eirós (Triacastela, Lugo). Historia Nova, IV: 9-29.

OlayA, V. 2014. Sistemas de Información Geográfica. 10.1017/CBO9781107415324.004

Ortega Martínez, P., \& Ruiz-Redondo, A. 2017. An approach for understanding site location preferences on Pas River Basin during Late Magdalenian.
Landscape analysis of Las Monedas cave. Journal of Archaeological Science: Reports, 0-1. 10.1016/ j.jasrep.2017.08.003

Parcero Oubiña, C., \& FÁbrega Álvarez, P. 2006. Diseño metodológico para el análisis locacional de asentamientos a través de un SIG de base «Raster». En $I$. Grau Mira (Ed.), La aplicación de los SIG en la arqueología del paisaje. Universidad de Alicante: 69-90.

Prieto, A., García-Rojas, M., Sánchez, A., Calvo, A., Domínguez-Ballesteros, E., Ordoño, J., \& GARCía-Collado, I. 2016. Stones in Motion: Cost units to understand flint procurement strategies during the Upper Palaeolithic in the south-western pyrenees using GIS. Journal of Lithic Studies, 3(1).

R CORE TEAm. 2015. R: A lenguage and environment for statistical computing. Vienna (Austria). Recuperado de http://www.r-project.org

RAmil Rego, E. 1989/1990. Habitabilidad cavernícola: elección de asentamientos. Brigantium, 6: 191-197.

Ramil Rego, E., \& Ramil Soneira, J. M. 1996. El fin de los tiempos glaciares en Galicia. Magdaleniense y Epipaleolítico. En: R. Fábregas Valcarce (Ed.), Os primeiros poboadores de Galicia: o Paleolítico, Sada (A Coruña), Edicios do Castro: 117-146.

Rey-Rodríguez, I., LóPez-García, J. M., Bennàsar, M., Bañuls-Cardona, S., Blain, H. A., BlancoLapaz, A., Rodríguez-Álvarez, X. P., DE Lombera-Hermida, A., Díaz-Rodríguez, M., AmeiJenDA-Iglesias, A., Agustí, J., \& FÁbregasVAlCARCE, R. 2016. Last Neanderthals and first Anatomically Modern Humans in the NW Iberian Peninsula: Climatic and environmental conditions inferred from the Cova Eirós small-vertebrate assemblage during MIS 3. Quaternary Science Reviews, 151: 185-197. 10.1016 j.quascirev.2016.08.030

Rios-Garaizar, J., \& García Moreno, A. 2015. Middle Paleolithic Mobility Patterns and Settlement System Variability in the Eastern Cantabrian Region (Iberian Peninsula): A GIS-Based Resource Patching Model. En N. J. Conard \& A. Delagnes (Eds.), Settlement Dynamics of the Middle Paleolithic and Middle Stone Age: 329-360.

Rodríguez Álvarez, X. P., Fábregas Valcarce, R. LazuÉn Fernández, T., DE LOMBera Hermida, A., Pérez Alberti, A., Peña, J. A., Rodríguez Rellán, C., Terradillos, M., Ameijenda, A. \& RoDríGuEz Álvarez, E. 2008. Nuevos yacimientos paleolíticos en la Depresión de Monforte de Lemos (Lugo, Galicia, España). Cuaternario y Geomorfologia, 22:71-92. 10.1016/S0034-7094 (11)70016-2

SÁnchez, A., Domínguez-Ballesteros, E., García-Rojas, M., Prieto, A., Calvo, A., \& Ordoño, J. 2016. Patrones de aprovisionamiento de sílex de las comunidades superopaleolíticas del Pirineo occidental: el "coste" como medida de análisis a partir de los SIG. Munibe Antropologia-Arkeologia, 67(6): 235-252. 10.21630/maa.2016.67.mis02

Steelman, K. L., de Lombera Hermida, A., Viñas Vallverdú, R., Rodríguez Álvarez, X. P., Carrera Ramírez, F., Rubio Mora, A., \& Fábregas ValCARCE, R. 2017. Cova Eirós: An integrated approach to dating the earliest known cave art in NW Iberia. Radiocarbon, 59(1), 151-164. https:// doi.org/10.1017/RDC.2017.4

Turrero, P., Domínguez-Cuesta, M. J., JimÉneZSÁnchez, M., \& García-VÁzquez, E. 2013. The spatial distribution of Palaeolithic human settle- 
Mikel Diaz-Rodriguez, Ramón Fábregas-Valcarce, Arturo de Lombera-Hermida \& Xosé Pedro Rodriguez-Álvarez

ments and its influence on palaeoecological studies: A case from Northern Iberia. Journal of Archaeological Science, 40(12): 4127-4138. 10.1016/ j.jas.2013.06.003

Valverde Tejedor, I. 2019. Patrones de aprovechamiento faunístico y estrategias de subsistencia durante la Transición Paleolítico medio-superior en el NO Peninsular. Tesis Doctoral, Universidade de Santiago de Compostela.

Viana-Soto, A. \& Pérez-Alberti, A. 2019. Periglacial deposits as indicators of paleotemperatures. A case study in the Iberian Peninsula: The mountains of Galicia. Permafrost and Periglacial Processes, 30 (4): 1-15. 10.1002/ppp.2026

Villar Quinteiro, R. 1996. El Paleolítico superior y el Epipaleolítico en Galicia. Tesis Doctoral, Universidade de Santiago de Compostela. 